\title{
The star formation region NGC 6530: Distance, ages and initial mass function $\star, \star \star$
}

\author{
L. Prisinzano ${ }^{1,2}$, F. Damiani ${ }^{2}$, G. Micela ${ }^{2}$, and S. Sciortino ${ }^{2}$ \\ 1 Dipartimento di Scienze Fisiche ed Astronomiche, Università di Palermo, Piazza del Parlamento 1, 90134 Palermo, Italy \\ e-mail: loredana@astropa.unipa.it \\ 2 INAF - Osservatorio Astronomico di Palermo, Piazza del Parlamento 1, 90134 Palermo, Italy
}

Received 12 March 2004 / Accepted 28 September 2004

\begin{abstract}
We present astrometry and $B V I$ photometry, down to $V \simeq 22$, of the very young open cluster NGC 6530, obtained from observations taken with the Wide Field Imager camera at the MPG/ESO $2.2 \mathrm{~m}$ Telescope. Both the $V$ vs. $B-V$ and the $V$ vs. $V-I$ color-magnitude diagrams (CMD) show that the upper main sequence is dominated by very bright cluster stars, while, because of the high obscuration of the giant molecular cloud surrounding the cluster, the blue envelopes of the diagrams at $V \gtrsim 14$ are limited to the main sequence stars at the distance of NGC 6530. This particular structure of the NGC 6530 CMD allows us to conclude that its distance is about $d \simeq 1250 \mathrm{pc}$, significantly lower than the previous determination of $d=1800 \mathrm{pc}$. We have positionally matched our optical catalog with the list of X-ray sources found in a Chandra-ACIS observation, finding a total of 828 common stars, $90 \%$ of which are pre-main sequence stars in NGC 6530. Using evolutionary tracks of Siess et al. (2000), mass and age values are inferred for these stars. The median age of the cluster is about $2.3 \mathrm{Myr}$; in the mass range (0.6-4.0) $M_{\odot}$, the Initial Mass Function (IMF) shows a power law index $x=1.22 \pm 0.17$, consistent with both the Salpeter index (1.35), and with the index derived for other young clusters; towards smaller masses the IMF shows a peak and then it starts to decrease.
\end{abstract}

Key words. Galaxy: open clusters and association: individual: NGC 6530 - techniques: photometric - astrometry stars: pre-main sequence stars - stars: Hertzprung-Russel (HR) and C-M diagrams

\section{Introduction}

Star formation regions and very young open clusters are crucial systems for understanding the star formation process because they allow us to derive Initial Mass Functions that are not affected by stellar and/or dynamical evolution effects. NGC 6530 $(l=6.14, b=-1.38)$ is an example of a very young open cluster located in front of the M 8 giant molecular cloud, also referred to as Lagoon Nebula (Lada et al. 1976). The brightest part of M 8 is the Hourglass Nebula, illuminated by the extremely young $\mathrm{O}$ star Herschel $36(\mathrm{O} 7 \mathrm{~V})$. Other stars of spectral type O, $9 \mathrm{Sgr}(\mathrm{O} 4 \mathrm{~V})$ and the binary system HD 165052 (O6.5 V + O6.5 V) excite the Lagoon Nebula. The open cluster NGC 6530 is located about 9!.8 eastwards of the Hourglass Nebula (corresponding to a projected distance from the Nebula of $3.6 \mathrm{pc}$, using the cluster distance $d=1250 \mathrm{pc}$ estimated in this work) and can be recognized by its brightest stars.

* Based on observations made with the European Southern Observatory telescopes obtained from the ESO/ST-ECF Science Archive Facility.

$\star \star$ Full Tables 4 and 5 are only available in electronic form at the CDS via anonymous ftp to

cdsarc.u-strasbg.fr $(130.79 .128 .5)$ or via

http://cdsweb.u-strasbg.fr/cgi-bin/qcat? J/A+A/430/941
Since the work of Walker (1957), several investigations have been devoted to study this cluster and to estimate its parameters. Using mainly photoelectric and photographic observations, many attempts have been made to estimate the distance of NGC 6530 from the CMDs, which show a normal cluster main sequence down to about A0 stars; fainter stars lie above the main sequence, indicating that the cluster is so young that its low mass members are still gravitationally contracting (Walker 1957). The lack of the complete cluster main sequence made it hard to obtain reliable estimates of the cluster distance. The NGC 6530 distance has been estimated by several authors to be in the range (1300-2000) pc (cf. Table 1), by taking advantage of spectroscopic observations to determine spectral types, and proper motions to select cluster members.

On the contrary, $U B V$ observations have allowed several authors to study the reddening law and to derive cluster reddening values very similar to the average value $E(B-V)=0.35$ recently derived by Sung et al. (2000), who have assumed a foreground reddening value, $E(B-V)_{\mathrm{fg}}=0.17$, as estimated by McCall et al. (1990). Finally, the age of the cluster was estimated to be in the range (1.5-2.0) Myr by several authors (van Altena \& Jones 1972; Sagar \& Joshi 1978; Sung et al. 2000), while Damiani et al. (2004) give a median age of 0.8 Myr. Because of the young age of this cluster, 
Table 1. Literature distance values for NGC 6530.

\begin{tabular}{ccc}
\hline \hline Distance $(\mathrm{pc})$ & $(m-M)_{0}$ & Reference \\
\hline $1380-2000$ & $10.7-11.5$ & Walker (1957) \\
1300 & 10.6 & The (1960) \\
1400 & 10.7 & Walker (1961) \\
1580 & 11.0 & Hiltner et al. (1965) \\
1780 & 11.25 & van Altena \& Jones (1972) \\
1380 & 10.7 & Kilambi (1977) \\
1820 & 11.3 & Sagar \& Joshi (1978) \\
1900 & 11.4 & Chini \& Neckel (1981) \\
1860 & 11.35 & McCall et al. (1990) \\
$1600-2000$ & $11.0-11.5$ & van den Ancker et al. (1997) \\
1400 & 10.74 & Loktin et al. (1997) \\
1800 & 11.25 & Sung et al. (2000) \\
$560-711$ & $8.75-9.26$ & Loktin \& Beshenov (2001) \\
\hline
\end{tabular}

low mass stars are expected to be still in the pre-main sequence phase, as already found in Sung et al. (2000) from photometric data down to the limiting magnitude $V \sim 17.119 \mathrm{X}$-ray point sources in the Lagoon Nebula region have been recently detected by Rauw et al. (2002) in a 20 ks XMM-Newton observation; they found that most of the X-ray sources are associated with pre-main sequence stars of low and intermediate mass. However, a larger list of point sources in the same region, with a much better spatial resolution, has recently been obtained by Damiani et al. (2004), using Chandra ACIS-I X-ray data. Damiani et al. show that their source sample is made up for least $90 \%$ by cluster members and that out of the $884 \mathrm{X}$-ray sources found, only 220 have a counterpart in the Sung et al. (2000) optical catalog and most of the latter are pre-main sequence members of NGC 6530. As suggested by Damiani et al., the remaining $\mathrm{X}$-ray sources are likely stars with magnitudes fainter than $V \sim 17$. The lack of published photometric data at magnitudes fainter than $V \sim 17$ has motivated the analysis of deep optical images to study the low mass stellar population in the NGC 6530 field and, in particular, the low-mass pre-main sequence stars of the cluster by means of cross-correlation with available X-ray data.

In this paper, we first present the observations and the data reduction procedure (Sect. 2) and the CMDs obtained using the optical catalog (Sect. 3). Next, we present the crosscorrelation of optical and X-ray data, from which membership is determined (Sect. 4), and the cross-correlation of optical and 2MASS IR data (Sect. 5); mass and age determination of cluster members and the analysis of the spatial distribution are discussed in Sect. 6. Finally, the Luminosity and the Initial Mass Functions (Sect. 7) and our conclusions (Sect. 8) are presented.

\section{The observational data}

The data used in this work come from the combination of optical BVI images taken with the Wide Field Imager (WFI) camera at the $2.2 \mathrm{~m}$ Telescope of the European Southern Observatory (ESO), a 60 ks Chandra ACIS X-ray observation
(Damiani et al. 2004) and public near-infrared data from the All-Sky Catalog of Point Sources of the Two Micron All Sky Survey (2MASS) $)^{1}$ (Cutri et al. 2003) available on the $\mathrm{WEB}^{2}$.

\subsection{Optical observations and data reduction}

The optical observations, consisting of 9 BVI images of NGC 6530, were taken using the WFI camera mounted at the Cassegrain focus of the ESO $2.2 \mathrm{~m}$ Telescope at La Silla (Chile). This instrument consists of a $4 \times 2$ mosaic of CCDs of $2048 \times 4096$ square pixels with a scale of 0.238 arcsec/pixel; each chip is $8.12 \times 16 ! 25$, while the full field of view (FOV) is $34 \times 33$ square arcmin. The observations, retrieved from the ESO/ST-ECF Science Archive Facility, are part of the ESO Imaging Survey (EIS) in the context of the PRE-FLAMES program (Momany et al. 2001). Details of the observations are given in Table 2 . The frames were taken under slightly variable seeing conditions with an improvement during the night of the Full Width Half Maximum (FWHM) of the point-spread function (PSF) from 1.76 to 0.83 arcsec, as measured on the frames.

Figure 1 shows the image obtained from the combination of the two deep dithered $I$ band images of the region around NGC 6530, corrected for the instrumental signatures as described below. The $F W H M$ of the PSF in this frame is about 0.83 arcsec. The very young cluster NGC 6530 can be recognized by its brightest stars located about 9'.8 eastwards of the O star Herschel 36, embedded in the Hourglass Nebula. The image shows regions of high and low stellar surface density according to the irregular pattern of the molecular cloud absorption.

The first stage of the data reduction process was the instrumental calibration of the images through the mscred package, a mosaic specific task implemented as an IRAF $^{3}$ package for the $\mathrm{NOAO}^{4}$ Mosaic Data Handling System. First the instrumental electronic bias of the images was subtracted using the overscan region and then the images were trimmed to remove the overscan region. Flat fielding for each filter was performed using a set of sky flat fields scaled to the median value of all chips combined into a master flat using the mscred flatcombine task.

A special treatment was required for the images in the $I$ band, affected by strong effects of fringing. To remove this instrumental artifact, the fringing pattern, provided by the MPG/ESO $2.2 \mathrm{~m}$ Telescope team ${ }^{5}$, was subtracted, scaling it to each exposure.

Detection of sources recorded in the analyzed digital frames was obtained using the DAOPHOT II/ALLSTAR

\footnotetext{
${ }^{1}$ A joint project of the University of Massachusetts and the Infrared Processing and Analysis Center/California Institute of Technology, funded by the National Aeronautics and Space Administration and the National Science Foundation.

${ }^{2}$ http://irsa.ipac.caltech.edu/

${ }^{3}$ Image Reduction and Analysis Facility.

${ }^{4}$ National Optical Astronomy Observatory.

5 Available at http://www.ls.eso.org/lasilla/Telescopes/ 2p2T/E2p2M/WFI
} 
Table 2. Log-book of the optical observations.

\begin{tabular}{|c|c|c|c|c|c|c|c|}
\hline $\begin{array}{c}\text { Target } \\
\text { EIS name }\end{array}$ & $\begin{array}{c}\text { RA (J2000) } \\
\quad(\mathrm{h} \mathrm{m} \mathrm{s})\end{array}$ & $\begin{array}{c}\operatorname{Dec}(\mathrm{J} 2000) \\
(\mathrm{d} \mathrm{m} \mathrm{s})\end{array}$ & Night & Filter & $\begin{array}{c}\text { Exp. Time } \\
{[\mathrm{s}]}\end{array}$ & $\begin{array}{c}\text { seeing } \\
F W H M\left[{ }^{\prime \prime}\right]\end{array}$ & Airmass \\
\hline NGC 6530 & 180448.0 & -241941.0 & 27-28 Jul. 2000 & $B$ & $1 \times 30+2 \times 240$ & $1.36-1.76$ & $1.268-1.236$ \\
\hline \multirow[t]{2}{*}{ (OC31) } & $"$ & $"$ & $"$ & V & $1 \times 30+2 \times 240$ & $1.19-1.36$ & $1.216-1.194$ \\
\hline & $"$ & $"$ & $"$ & $I$ & $1 \times 30+2 \times 240$ & $0.83-1.05$ & $1.175-1.154$ \\
\hline
\end{tabular}

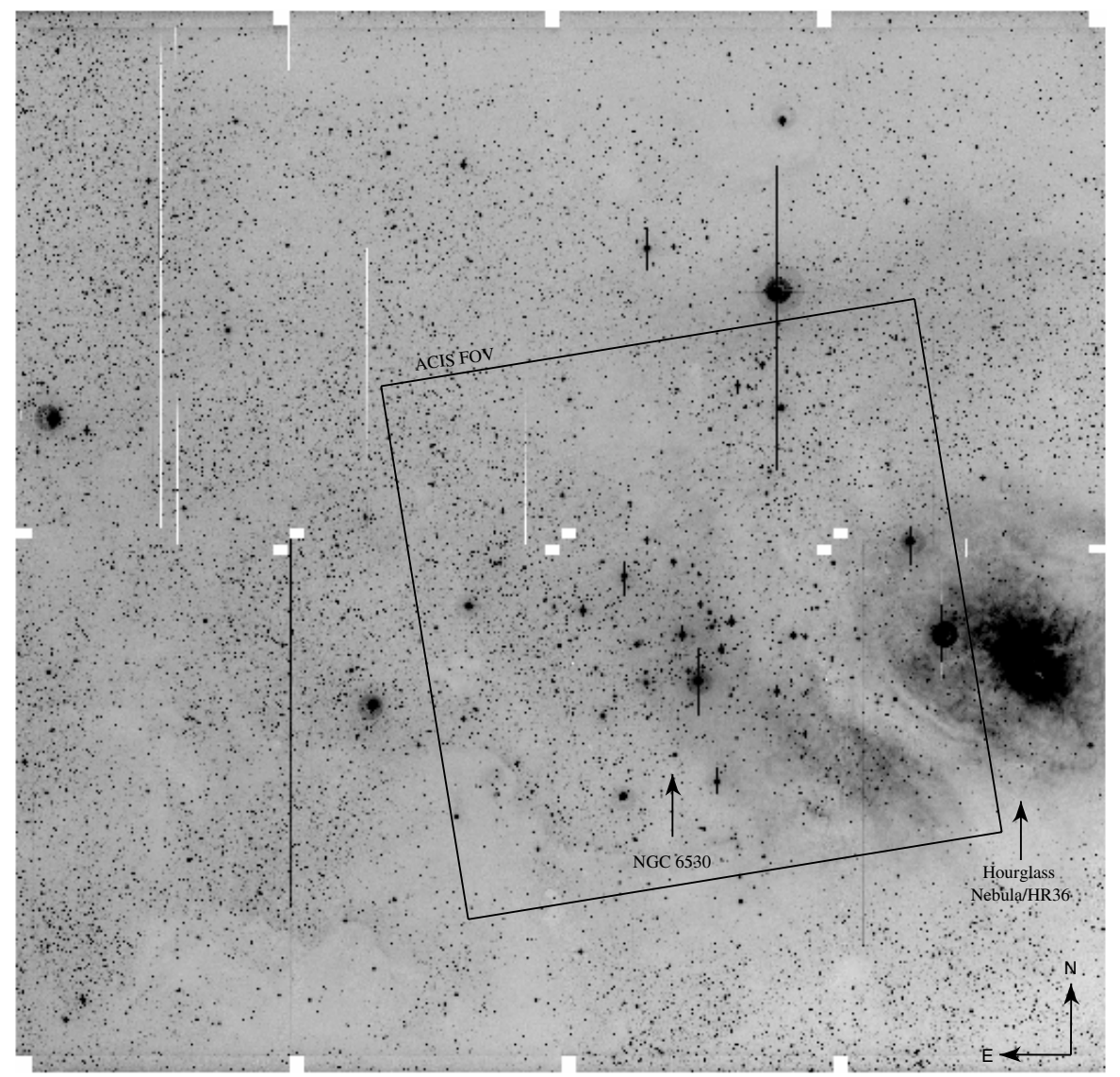

Fig. 1. Image obtained from the combination of the two deep dithered $I$ band images of the region around NGC 6530 observed with the WFI. White squares are unexposed regions due to the detector gaps.

photometric routines described in Stetson (1987). A more accurate PSF fitting photometry was performed by submitting all images to the routine ALLFRAME (Stetson 1994) which simultaneously uses the geometric and photometric information from all images to derive a self-consistent set of positions and magnitudes for all detected sources.

In the present case, all images are characterized by a very strong sky background gradient and thus particular attention was devoted to estimating the minimum sky level (DAOPHOT parameter LOW GOOD DATUM $=50$ in units of standard deviations) in order to include legitimate sky pixels where the background is faint.

A non-standard procedure, specifically suited for this case, was applied to the chip containing the star Herschel 36 because a significant number of spurious detections was found due to the emission from the nearby Hourglass Nebula. In order to recognize true star detections, the list of the stars released by ALLSTAR was filtered according to the sharp parameter. After this selection was applied, the simultaneous PSF fitting photometry was performed with the routine ALLFRAME, which releases a star-subtracted image from each frame. The star-subtracted images were used to obtain a median image, which was then smoothed with a Gaussian smoothing $\sigma$ larger than the FWHM of the PSF but smaller than the angular scale of the structures in the nebula. Finally a median image without nebula was obtained by subtracting the smoothed starsubtracted median image from the median image obtained from the original images. A more reliable star list, determined using the latter median nebula-subtracted image, was given as input to ALLFRAME to obtain the final PSF fitting photometry.

In order to convert the profile-fitting photometry to the standard photometric system, a magnitude zero point was calculated for each chip. This was done using the growth curve method described in Stetson (1990). First, we selected the 
Table 3. Coefficients of the transformation to the standard system for each filter and averaged over all chips.

\begin{tabular}{cccc}
\hline \hline Filter & Av. zero point & Extinction & Av. color term \\
\hline$V$ & $0.905 \pm 0.021$ & 0.14 & $0.066 \pm 0.046$ \\
$B$ & $0.528 \pm 0.030$ & 0.25 & $-0.245 \pm 0.019$ \\
$I$ & $1.904 \pm 0.009$ & 0.09 & $-0.133 \pm 0.034$ \\
\hline
\end{tabular}

5268 stars used to define the PSF model; next, all other objects were removed from the frames and aperture photometry was carried out at different radii. The DAOGROW code (Stetson 1990) was used to derive growth curves and COLLECT was used to calculate the "aperture correction" coefficient for each chip, from the difference between PSF-fitting magnitudes and aperture photometry magnitudes of the selected stars.

The procedure described above was also applied to a set of images of the Landolt (1992) standard fields SA92 and SA107 obtained with WFI/2.2 m during the same night.

Using the $v, b$ and $i$ instrumental magnitudes and the $V$, $B$ and $I$ magnitudes of the Johnson-Kron-Cousins photometric system of the standard stars falling in these fields, the transformation coefficients to the standard system were performed using the following equations:

$$
\begin{aligned}
v & =V+A_{0}+A_{1} \times X+A_{2} \times(V-I), \\
b & =B+B_{0}+B_{1} \times X+B_{2} \times(B-V), \\
i & =I+C_{0}+C_{1} \times X+C_{2} \times(V-I) .
\end{aligned}
$$

Where $X$ is the airmass and $A_{0}, B_{0}$ and $C_{0}$ are the magnitude zero points, $A_{1}, B_{1}$ and $C_{1}$ are the extinction coefficients, and $A_{2}, B_{2}$ and $C_{2}$ are the color terms. The list of standard stars used to determine the coefficients contains the standard stars defined in the Landolt (1992) catalog and a number of secondary standard stars present in the Landolt fields and defined by Stetson (2000). Of these stars, we have between 25 and 161 measurements for each chip, except for chip \# 53 (the north-western chip in Fig. 1) because neither Landolt's catalogue standards nor Stetson's catalogue standards fell in this chip. Since the observations of the standard stars do not cover a wide airmass range, we were not able to derive the extinction coefficients. The extinction coefficients typical of La Silla (Stetson, private communication) were adopted, and the zero point and the color term were calculated for all chips except for chip \# 53. Stars with magnitude residual values above the $3 \sigma$ level were not considered for the coefficient fitting. Considering the homogeneous characteristic of the instrument, we chose to compute new zero points fixing the color term to the mean value of the color terms of the chips \# 50, \#51, \#54, \#55, \#56 for which the number of measured standard stars was larger than 40. Differences smaller than 0.1 mag between the zero points of different chips were found. Based on this latter result, we used their average and the above determined average color term to calibrate the stars in chip \# 53, where no standard stars are found. The resulting coefficients are given in Table 3.

Using these coefficients and the inverted form of Eqs. (1), the $B, V, I$ magnitudes of all objects detected in at least two filters were calculated. In order to test the self-consistency of the photometry, we compared the photometry of the stars common to contiguous chips. Common stars between chips were found because the deep mosaic images were dithered by about 30 arcsec in RA and about 60 arcsec in Dec in order to cover the inter-chip gaps. By matching contiguous chips we found about 10-30 common stars per chip pair for which we compared the photometry. We found mean offsets in the photometric zero points $\$ 4 \%$ in $V$ and $I$, and $\$ 7 \%$ in $B$; this supports the choice to use the average zero point of the other chips to calibrate the data in chip \# 53.

In order to consider only stellar-like objects, a data selection was applied by including only objects with sharp between -0.5 and 0.5 (Stetson 1987) i.e. objects with a brightness distribution consistent with a point source. After this selection, our catalog includes 53581 objects.

\subsubsection{Astrometry}

Celestial coordinates of the detected optical stars were obtained using the Guide Star Catalogue Version 2.2.01 (GSC 2.2, STScI, 2001) as reference catalogue. The first step was to match the list of celestial coordinates of stars retrieved from the GSC 2.2 catalog, with the list of pixel coordinates obtained for each chip as described in the previous section. The transformation between the two systems was obtained by applying the appropriate projection to the celestial coordinates and a linear transformation to the pixel coordinates. The initial estimate for the linear transformation is determined using three stars whose celestial and pixel coordinates are both known. To find the best astrometric solution, we matched the two catalogs using a conservative matching tolerance of 0 '. 3 . With this matching tolerance 2671 common stars were found (IRAF task ccxymatch) from a total of 33554 sources (appearing in at least $50 \%$ of our frames) used to match the GSC 2.2 catalog. The matched lists of pixel and celestial coordinates of each chip were used to compute the plate solution. The sky projection was obtained using a combination of the tangent plate projection and polynomials (IRAF task ccmap). Finally, using the derived astrometric solution, we computed celestial coordinates of our photometric catalog stars.

In order to estimate our astrometric accuracy we matched stars of our catalog with $V<20$ with the GSC 2.2 catalog, used as reference to find the astrometric solution, and considered the offset distribution within a relatively large value $\left(5^{\prime \prime}\right)$. From this distribution we subtracted the expected distribution of spurious identifications, obtaining the distribution of true identifications only. The resulting rms is 0 . $^{\prime} 4$, which is therefore our final accuracy.

\subsubsection{Comparison with previous catalogs}

As an external check of our photometry we selected stars in our catalog with $V<17.5$ and compared them with the Sung et al. (2000) catalog limited to $V \leq 17$. By matching the two catalogs, we found a systematic offset in both right ascension and declination of $\sim-11^{\prime \prime} 05$ and $\sim 0$.'34, respectively. 
To estimate the appropriate matching radius for comparing the two catalogs, we first removed the coordinate offsets and then considered the offset distribution within $7^{\prime \prime}$. We found that the rms width of the distribution is 0.5 and that the contribution of spurious identifications becomes dominant at offsets larger than $1^{\prime \prime}$.

With the aim to include only reliable matches between our and the Sung et al. catalogs, we adopted a matching radius of 0.5 , finding 576 stars with offsets $-0.01 \pm 0.30$ and $-0 .{ }^{\prime} 02 \pm 0.22$, in RA and Dec, respectively. The mean offsets and the rms of the photometric residuals are $0.006 \pm$ $0.060 \mathrm{mag}, 0.007 \pm 0.075 \mathrm{mag}$ and $0.037 \pm 0.055 \mathrm{mag}$ in $V$, $(B-V)$, and $(V-I)$, respectively. These values are computed after applying a $3 \sigma$ clipping to the photometric residuals.

Finally, we compared our photometric catalog with the catalog of Walker (1957) and Kilambi (1977) consisting of a total of 319 stars. Of these stars, only the 150 stars with $V>9.5$, falling in the WFI FOV and with celestial coordinates found and retrieved from the SIMBAD database, were considered. With a matching radius of $9^{\prime \prime}$ we found 131 stars and a number of mis-identifications that we discarded based on their photometric residuals. By considering the stars having both $B$ and $V$ magnitudes in the Walker (1957) and Kilambi (1977) catalog, we found $\Delta V=0.002 \pm 0.073$ and $\Delta(B-V)=$ $0.006 \pm 0.081$, after applying a $5 \sigma$ clipping to the photometric residuals. The remaining 19 stars with no counterpart in our catalog have $V \sim 10$, hence they are saturated in the WFI frames.

All previous comparisons show that our magnitudes and colors are in excellent agreement with those measured by other authors. Table 4 shows the results of such comparisons; Cols. 1-9 list the sequential number, celestial coordinates and photometry of our catalog; the star number used by Sung et al. (2000, denoted as $\left.I D_{S C B}\right)$ and the astrometric and photometric residuals obtained from the comparison with the Sung et al. (2000) catalog are given in Cols. 10-15; the star number used by Walker (1957) and Kilambi (1977, denoted as $\left.I D_{W K}\right)$ and the astrometric and photometric residuals obtained from the comparison with the Walker (1957) and Kilambi (1977) catalogs are given in Cols. 16-20.

\subsection{X-ray and near-infrared data}

To better understand part of our optical data we used a list of X-ray sources, presented in Damiani et al. (2004). These data were obtained from a $60 \mathrm{ks}$ observation carried out on June 18-19, 2001 with the Chandra ACIS-I CCD detector having a very narrow PSF ( $F W H M \sim 0.5^{\prime \prime}$ on-axis). The observation consists of a FOV of $17^{\prime} \times 17^{\prime}$ pointed toward $\mathrm{RA}=18^{\mathrm{h}} 04^{\mathrm{m}} 24.38$, Dec $=-24^{\circ} 21^{\prime} 05^{\prime \prime} .8$, i.e. centered on the NGC 6530 cluster. 884 point-like X-ray sources were detected, as described in detail by Damiani et al. (2004).

In addition, to complement the optical data, we used $J H K_{\mathrm{S}}$ infrared (IR) photometry taken from the All-Sky Point Source public catalogue of the Two Micron All Sky Survey (2MASS) (Cutri et al. 2003).

\section{The color-magnitude diagrams}

\subsection{Optical data}

The final optical photometric catalog was obtained by adding to our data the stars of the Sung et al. (2000) catalog that were not detected in the WFI images. These are very bright stars ( $V \lesssim 10 \mathrm{mag}$ ), saturated in our images, or stars falling out of the WFI FOV or on the edges of the chips, for a total of $123 \mathrm{ob}-$ jects. To select these stars, we considered the stars of the Sung et al. (2000) catalog whose astrometric offset with respect to our catalog is larger than $1^{\prime \prime}$, the radius for which the number of matched stars drops to zero.

CMDs of all stars of the complete optical catalog were constructed after selecting the stars with errors in both the $V$ and $I$ magnitudes smaller than 0.2 mag; stars with larger (statistical) errors cannot be placed accurately on the CMD so their inclusion is meaningless. We applied this selection only to the $V$ and $I$ magnitudes because in the following analysis we mainly will use the $V$ vs. $V-I$ CMD. Nevertheless, the $V$ vs. $B-V$ diagram was obtained using stars of the cleaned catalog with errors in $B$ smaller than $0.2 \mathrm{mag}$. Using this selection criterion for our data we found that our detection limit is $V \sim 23$. The completeness of our data was determined via artificial star tests. A total of about 1650 artificial stars was added on each chip and photometry of the artificial frames was performed using the same reduction procedure applied to the original frames. In order to test the completeness of the data used in the following analysis, the results were obtained by considering the number of retrieved artificial stars with sharp between -0.5 and 0.5 and errors in the $V$ and $I$ magnitudes smaller than 0.2 mag. We found that our data are $100 \%$ complete above $V \sim 20$ and $I \sim 18.5$, while they are more than $50 \%$ complete above $V \sim 22$ and $I \sim 19.5$.

The $V$ vs. $B-V$ and the $V$ vs. $V-I$ CMDs, obtained using the cleaned catalogs, are shown in Fig. 2, where horizontal bars indicate the median errors in color, while vertical bars (barely visible), indicate the median errors in magnitude for one magnitude bins. The number of stars and the error bars in the $V$ vs. $B-V$ diagram are smaller than those in the $V$ vs. $V-I$ diagram because in the $V$ vs. $B-V$ the selection has been applied to the $B, V$ and $I$ magnitudes while in the $V$ vs. $V-I$ diagram it has been applied only to the $V$ and $I$ magnitudes.

The horizontal dashed line in each diagram, indicating the magnitude completeness limit of the Sung et al. (2000) catalog, gives an idea of the amount of new photometric information yielded by this survey.

Both diagrams clearly show the upper main sequence $(B-$ $V \lesssim 0.5$ and $V-I \lesssim 0.6$ ) dominated by the very bright cluster stars. These are the more massive stars which, having a very short pre-main sequence lifetime, have already reached the main sequence showing a very small age spread.

As already noted by Sung et al. (2000), a sequence of stars appears in the range $0.7 \lesssim V-I \lesssim 1.5$ and $13 \lesssim V \lesssim 18$. These stars were interpreted by Sung et al. (2000) as foreground stars less reddened than the remaining stars in the field. 
Table 4. Cross-identifications of this catalog with the previous works of Walker (1957), Kilambi (1977) and Sung et al. (2000). The complete table is available in electronic form at the CDS.

\begin{tabular}{|c|c|c|c|c|c|c|c|c|c|c|c|c|c|c|c|c|c|c|c|}
\hline ID & $\begin{array}{c}\mathrm{RA}(2000) \\
(\mathrm{h} \mathrm{m} \mathrm{s})\end{array}$ & $\begin{array}{c}\operatorname{Dec}(2000) \\
(\mathrm{d} \mathrm{m} \mathrm{s})\end{array}$ & $B$ & $\overline{\sigma_{B}}$ & $\bar{V}$ & $\overline{\sigma_{V}}$ & $I$ & $\sigma_{I}$ & $\mathrm{ID}_{S B C}$ & $\begin{array}{c}\Delta \mathrm{RA}^{a} \\
(\operatorname{arcsec})\end{array}$ & $\begin{array}{l}\Delta \operatorname{Dec}^{a} \\
(\operatorname{arcsec})\end{array}$ & $\Delta V^{a}$ & $\Delta(B-V)^{a}$ & $\Delta(V-I)^{a}$ & $I D_{\mathrm{WK}}$ & $\begin{array}{l}\Delta \mathrm{RA}^{b} \\
(\operatorname{arcsec})\end{array}$ & $\begin{array}{l}\Delta \operatorname{Dec}^{b} \\
(\operatorname{arcsec})\end{array}$ & $\overline{\Delta V^{b}}$ & $\Delta(B-V)^{b}$ \\
\hline WFI 5173 & 180435.45 & -243228.82 & 14.749 & 0.002 & 13.889 & 0.003 & 12.860 & 0.004 & 732 & 0.29982 & -0.36392 & -0.061 & 0.016 & 0.022 & & & & & \\
\hline WFI 5249 & 180445.36 & -243226.44 & 17.692 & 0.005 & 16.694 & 0.003 & 15.477 & 0.006 & 836 & 0.39976 & -0.69351 & -0.028 & -0.040 & 0.006 & & & & & \\
\hline WFI 5377 & 180433.15 & -243222.37 & 18.709 & 0.004 & 16.601 & 0.003 & 14.008 & 0.003 & 712 & 0.39976 & -0.39825 & -0.036 & 0.177 & 0.072 & & & & & \\
\hline WFI 5387 & 180413.26 & -243222.09 & 15.973 & 0.006 & 15.115 & 0.006 & 14.091 & 0.003 & 451 & 0.49970 & -0.34332 & -0.108 & -0.007 & 0.009 & & & & & \\
\hline WFI 5449 & 180431.27 & -243220.17 & 13.460 & 0.008 & 13.049 & 0.009 & 12.605 & 0.012 & 687 & 0.69958 & -0.32959 & -0.038 & 0.069 & 0.030 & 131 & 3.69780 & -2.16980 & -0.031 & 0.051 \\
\hline WFI 5472 & 180347.86 & -243219.58 & 17.853 & 0.003 & 16.789 & 0.003 & 15.587 & 0.006 & 207 & 0.29982 & -0.52872 & -0.080 & 0.028 & 0.024 & & & & & \\
\hline WFI 5483 & 180437.62 & -243219.21 & 15.493 & 0.023 & 14.305 & 0.012 & 12.811 & 0.031 & 756 & -0.19988 & -0.26779 & 0.001 & 0.181 & -0.021 & & & & & \\
\hline WFI 5493 & 180351.58 & -243218.72 & 15.619 & 0.003 & 14.779 & 0.001 & 13.778 & 0.004 & 247 & -0.69958 & -0.46005 & -0.061 & 0.035 & 0.024 & & & & & \\
\hline WFI 5556 & 180348.69 & -243216.89 & 15.556 & 0.002 & 14.596 & 0.003 & 13.327 & 0.005 & 218 & -0.49971 & -0.53558 & -0.107 & 0.124 & 0.030 & & & & & \\
\hline WFI 5660 & 180348.32 & -243213.42 & 14.556 & 0.005 & 13.645 & 0.003 & 12.635 & 0.010 & 212 & -0.19988 & -0.44632 & -0.069 & 0.033 & 0.017 & & & & & \\
\hline WFI 5828 & 180343.17 & -24327.96 & 18.018 & 0.003 & 16.018 & 0.005 & 13.681 & 0.006 & 168 & -0.69960 & -0.50125 & -0.031 & 0.129 & 0.102 & & & & & \\
\hline WFI 5857 & 180336.99 & -24327.15 & 17.187 & 0.003 & 14.814 & 0.004 & 11.861 & 0.010 & 117 & -0.49972 & -0.47379 & -0.005 & 0.143 & 0.129 & & & & & \\
\hline WFI 5875 & 180350.34 & -24326.68 & 19.092 & 0.007 & 16.951 & 0.005 & 14.451 & 0.003 & 234 & 0.00000 & -0.51498 & 0.021 & 0.091 & 0.114 & & & & & \\
\hline WFI 5965 & 180345.52 & -24323.92 & 18.660 & 0.004 & 16.605 & 0.003 & 14.090 & 0.005 & 187 & -0.09994 & -0.45319 & -0.049 & 0.128 & 0.083 & & & & & \\
\hline WFI 5979 & 180330.39 & -24323.45 & 17.355 & 0.011 & 16.313 & 0.007 & 15.024 & 0.016 & 83 & -0.59966 & -0.70038 & -0.141 & 0.045 & 0.031 & & & & & \\
\hline WFI 5995 & 180351.04 & -24323.01 & 17.879 & 0.005 & 15.416 & 0.002 & 12.456 & 0.013 & 241 & 0.19989 & -0.44632 & 0.028 & 0.122 & 0.102 & & & & & \\
\hline WFI 6004 & 180359.02 & -24322.75 & 16.633 & 0.002 & 15.602 & 0.002 & 14.406 & 0.003 & 303 & 0.00000 & -0.40512 & -0.034 & -0.027 & 0.037 & & & & & \\
\hline WFI 6057 & 180429.68 & -24320.99 & 19.260 & 0.006 & 16.643 & 0.004 & 13.365 & 0.004 & 675 & 0.79956 & -0.43945 & 0.071 & 0.072 & 0.140 & & & & & \\
\hline WFI 6149 & 180420.02 & -243157.73 & 17.911 & 0.002 & 16.289 & 0.006 & 14.262 & 0.002 & 541 & -0.29984 & -0.45319 & -0.005 & 0.004 & 0.058 & & & & & \\
\hline WFI 6278 & 180343.40 & -243152.64 & 18.538 & 0.003 & 16.515 & 0.004 & 14.071 & 0.005 & 172 & -0.19989 & -0.47379 & -0.049 & 0.064 & 0.077 & & & & & \\
\hline
\end{tabular}

$a$ SCB comparison.
${ }^{b}$ Walker-Kilambi comparison. 

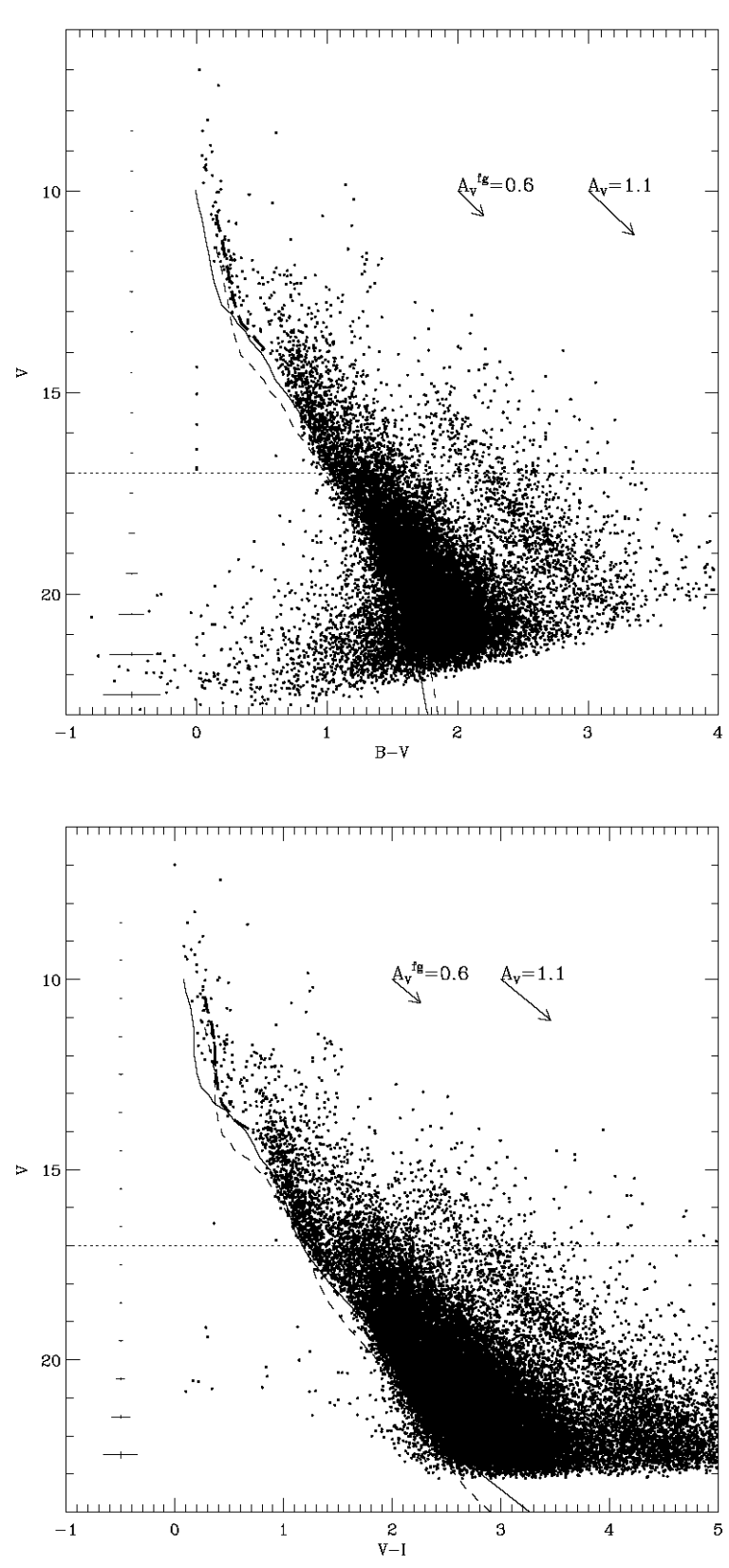

Fig. 2. The $V$ vs. $B-V$ and the $V$ vs. $V-I$ CMDs of selected stars (20 253 and 37553 , respectively) in the WFI FOV. Horizontal bars indicate the median errors in color, while vertical bars (barely visible) indicate the median errors in magnitude for bins of one magnitude. The horizontal dashed line in each diagram indicates the magnitude completeness limit of the Sung et al. (2000) catalog. The solid line is the Zero Age Main Sequence (ZAMS) of Siess et al. (2000) assuming a cluster distance $d=1250 \mathrm{pc}$ and $E(B-V)=0.20$; the thick long dashed line is the same ZAMS at the same distance but with the average cluster reddening $E(B-V)=0.35$ estimated by Sung et al. (2000); finally the dashed line is the ZAMS of Sung \& Bessell (1999) at a distance of $1800 \mathrm{pc}$ and the cluster reddening $E(B-V)=0.35$, derived by Sung et al. (2000). The extinction vectors $A_{\mathrm{V}}^{\mathrm{fg}}=0.6$ and $A_{\mathrm{V}}=1.1$ correspond to reddening $E(B-V)=0.20$ and $E(B-V)=0.35$, adopted for foreground and cluster stars, respectively.

Cluster stars fainter than $V \simeq 14$ are expected to be premain sequence stars, which populate a large region in the CMDs, highly contaminated by field stars.
One of the most important features of these diagrams is the well defined blue envelope; it is due to the presence of the giant molecular cloud, which prevents us from seeing field stars (mostly main-sequence) more distant than the cloud. Therefore, the well defined blue envelope of the CMDs is populated by main sequence field stars at the distance of the cloud. Background field stars, i.e. stars more distant than the cloud, are highly obscured by the cloud and therefore they would be visible at magnitudes and colors much fainter and much redder than their intrinsic values.

Finally, we note the presence of an unexpected sequence of red stars, well separated from the bulk of the objects. The analysis of the spatial distribution of these stars indicates that they are uncorrelated with the region dominated by the cluster stars, identified by a clustering of selected members, as will be shown in Sect. 6. On the contrary, they cover almost uniformly the less obscured regions of the molecular cloud and are likely associated with clump red giants at larger distances. Since we are here mainly interested to study the stellar population of the very young cluster NGC 6530, we defer a further investigation of these stars to a future paper.

\subsubsection{Cluster distance}

As already mentioned in the previous section, at the age and distance of NGC 6530 only stars brighter than $V \sim 14$ define the main sequence, which in this magnitude range is almost vertical, preventing the estimate of the cluster distance using the usual photometric method.

Fainter cluster stars do not define any sequence, but, on the contrary, they populate a large region of the CMD which cannot be used to constrain the cluster distance.

Instead, we derived a reliable estimate of the cluster distance by taking advantage of the particular feature of the CMDs: the well defined blue envelope. It is populated by main sequence field stars at the cloud distance, which is very similar to the cluster distance if one makes the very reasonable assumption that the cluster lies just before the cloud. In fact, the blue envelope fixes a magnitude limit within which we see stars (either belonging to the cluster or not), with a reddening equal or less than the cluster reddening. In particular, stars along the blue envelope brighter than $V \sim 14$ are cluster stars that have reached the main sequence, while fainter stars along the blue envelope are foreground stars up to the cloud distance.

We superposed the theoretical ZAMS computed by Siess et al. (2000) on CMDs (solid line in Fig. 2) and we found that the best match between this curve and the blue envelope of the star distribution is found if a distance of $d \simeq 1250 \mathrm{pc}$ is adopted, corresponding to a distance modulus $\left(V-M_{\mathrm{V}}\right)_{0} \simeq 10.48$, and a reddening $E(B-V)=0.20$ (corresponding to $A_{\mathrm{V}}=0.6$ and $E(V-I)=0.26$ using the reddening law $E(V-I)=$ $1.3 \times E(B-V)$ by Munari \& Carraro 1996). We note that the reddening $E(B-V)=0.20$ is the value derived for foreground field stars, which suffer from a reddening significantly smaller than that of the cluster stars. In fact, for $V<14$, the theoretical model is bluer than the bright stars because these are main sequence cluster stars at the same distance $(d \simeq 1250 \mathrm{pc})$ but 
Table 5. Cross-identifications of the optical catalog with the X-ray source catalog of Damiani et al. (2004).

\begin{tabular}{cccccccccccc}
\hline \hline $\begin{array}{c}\text { RA(2000) } \\
(\mathrm{h} \mathrm{m} \mathrm{s})\end{array}$ & $\begin{array}{c}\text { Dec(2000) } \\
(\mathrm{d} \mathrm{m} \mathrm{s})\end{array}$ & Cat & ID & $B$ & $\sigma_{B}$ & $V$ & $\sigma_{V}$ & $I$ & $\sigma_{I}$ & $\begin{array}{c}\text { ID(X) } \\
\text { Mult. } \\
\#\end{array}$ \\
\hline 18448.38 & -24293.94 & WFI & 10889 & 17.346 & 0.008 & 15.901 & 0.008 & 14.011 & 0.005 & 811 & 1 \\
18426.49 & -24290.30 & WFI & 10947 & 20.717 & 0.109 & 18.975 & 0.024 & 16.096 & 0.031 & 528 & 2 \\
18426.46 & -242859.95 & WFI & 10956 & 18.314 & 0.019 & 16.729 & 0.011 & 14.771 & 0.007 & 528 & 2 \\
18430.41 & -242853.16 & WFI & 11091 & 20.006 & 0.010 & 18.289 & 0.018 & 15.930 & 0.004 & 609 & 1 \\
18426.29 & -242848.61 & WFI & 11173 & 20.225 & 0.013 & 18.809 & 0.011 & 16.370 & 0.004 & 524 & 1 \\
18421.80 & -242846.25 & WFI & 11216 & 22.255 & 0.065 & 20.345 & 0.024 & 16.760 & 0.007 & 417 & 1 \\
18431.37 & -242844.49 & WFI & 11246 & 21.783 & 0.046 & 19.634 & 0.034 & 16.536 & 0.004 & 628 & 1 \\
18422.54 & -242840.75 & WFI & 11308 & 15.464 & 0.003 & 14.705 & 0.004 & 13.693 & 0.005 & 432 & 1 \\
18416.76 & -242836.94 & WFI & 11376 & 11.803 & 0.038 & 11.580 & 0.003 & 11.102 & 0.005 & 305 & 1 \\
18423.34 & -242831.98 & WFI & 11471 & 19.082 & 0.018 & 17.726 & 0.004 & 15.938 & 0.004 & 451 & 1 \\
\hline
\end{tabular}

with reddening $E(B-V)=0.35$ (corresponding to $A_{\mathrm{V}}=1.1$ and $E(V-I)=0.46)$, which is the average cluster reddening given by Sung et al. (2000), as shown by the thick long dashed line in Fig. 2. For comparison the ZAMS at the distance of 1800 pc, i.e. the distance adopted by Sung et al. (2000), is shown as a dashed line; the extinction vectors $A_{\mathrm{V}}^{\mathrm{fg}}=0.6$ and $A_{\mathrm{V}}=1.1$, corresponding to foreground stars and cluster stars respectively, are also shown in Fig. 2.

The distance value $d \simeq 1250 \mathrm{pc}$ derived for NGC 6530 is very close to the lower limit of the wide range of values (1300-2000 pc) photometrically determined for bright stars in previous works; it is, instead, significantly larger than the value obtained by Loktin \& Beshenov (2001) from the Hipparcos trigonometric parallaxes of 7 stars (see Table 1).

\section{Optical data and X-ray source identifications}

As already discussed in the introduction, X-ray observations are very useful tracers of membership in star formation regions or very young clusters (e.g. Alcala et al. 1995; Randich et al. 1995; Sterzik et al. 1995; Flaccomio et al. 2000), such as NGC 6530. Alternative membership assessment methods are not suitable for NGC 6530, since neither reliable proper motion measures nor radial velocities for magnitudes fainter than $V \sim 14$ are yet available.

To trace the pre-main sequence locus of NGC 6530, the optical data derived in this work were matched with X-ray sources published by Damiani et al. (2004), although the Chandra ACIS FOV is about 4 times smaller than the WFI FOV. However, the Chandra ACIS FOV is centered very close to the NGC 6530 nominal center RA $=18^{\mathrm{h}} 04^{\mathrm{m}} 25^{\mathrm{s}}$, Dec $=$ $-24^{\circ} 22^{\prime} 00^{\prime \prime}$ (Damiani et al. 2004), thus allowing us to study most of the cluster stars.

The total number of optical sources falling in the Chandra ACIS FOV is 8956, while the Damiani et al. (2004) catalog contains $884 \mathrm{X}$-ray sources. In order to estimate the number of X-ray sources that are not cluster members, Damiani et al. (2004) examined an observation of the Galactic plane obtained with the same detector and used it as "control field". By analyzing the detected count-rate distributions of the two fields they conclude that at least $90 \%$ of the X-ray sources are very probable cluster members.

To cross-correlate the X-ray and optical catalogs we used a matching distance $d<4 \sigma_{\mathrm{X}}$ (where $\sigma_{\mathrm{X}}$ is the X-ray position error, see Damiani et al. 2004). In doing this, we found and corrected a systematic shift between X-ray and WFI positions of 0.2 in RA, and - 0.26 in Dec. For three X-ray sources with such a small X-ray error that $4 \sigma_{\mathrm{X}}$ was less than $1^{\prime \prime}$.5 we relaxed the identification condition to $d<1$ '"5. This resulted in a number of multiple identifications, among which four turned into single identifications when we used a reduced distance $d<1.5 \sigma_{\mathrm{X}}$. This leaves us with 721 single, 44 double and 3 triple X-ray identifications in the optical catalog; in addition, one X-ray source has 4 optical identifications and one further $\mathrm{X}$-ray source has 6 optical identifications. These multiple identifications are near the edge of the ACIS FOV where the spatial resolution is much worse than in the center. The total number of X-ray sources with WFI counterpart(s) is therefore 770; of them only $15 \mathrm{X}$-ray identified stars come from the Sung et al. catalog and are not in the WFI catalog. The total number of optical sources with an X-ray counterpart is 828. The agreement between X-ray and WFI positions is excellent in most cases, with offsets below $1^{\prime \prime}$. The final list, comprising 770 X-ray sources, with their optical counterpart(s), is given in Table $5^{6}$, where Cols. 1 and 2 are the celestial coordinates, Col. 3 indicates the parent optical catalog, Col. 4 is the optical identification number, Cols. 5-10 are the BVI magnitudes and their uncertainties, Col. 11 is the X-ray identification number of the Damiani et al. (2004) catalog and, finally, Col. 12 is the number of optical sources corresponding to the X-ray detections.

Figure 3 shows the $V$ vs. $V-I$ CMD of stars falling in the Chandra ACIS FOV. Dots indicate all stars in the optical catalog falling in the ACIS FOV, while large filled symbols indicate optical stars having an X-ray counterpart; stars added from the Sung et al. (2000) catalog are marked by squares. As expected, $\mathrm{X}$-ray detected stars trace very well the CMD region occupied by the cluster stars. The cluster region identified by 220 X-ray

\footnotetext{
${ }^{6}$ Available in electronic form at the CDS.
} 


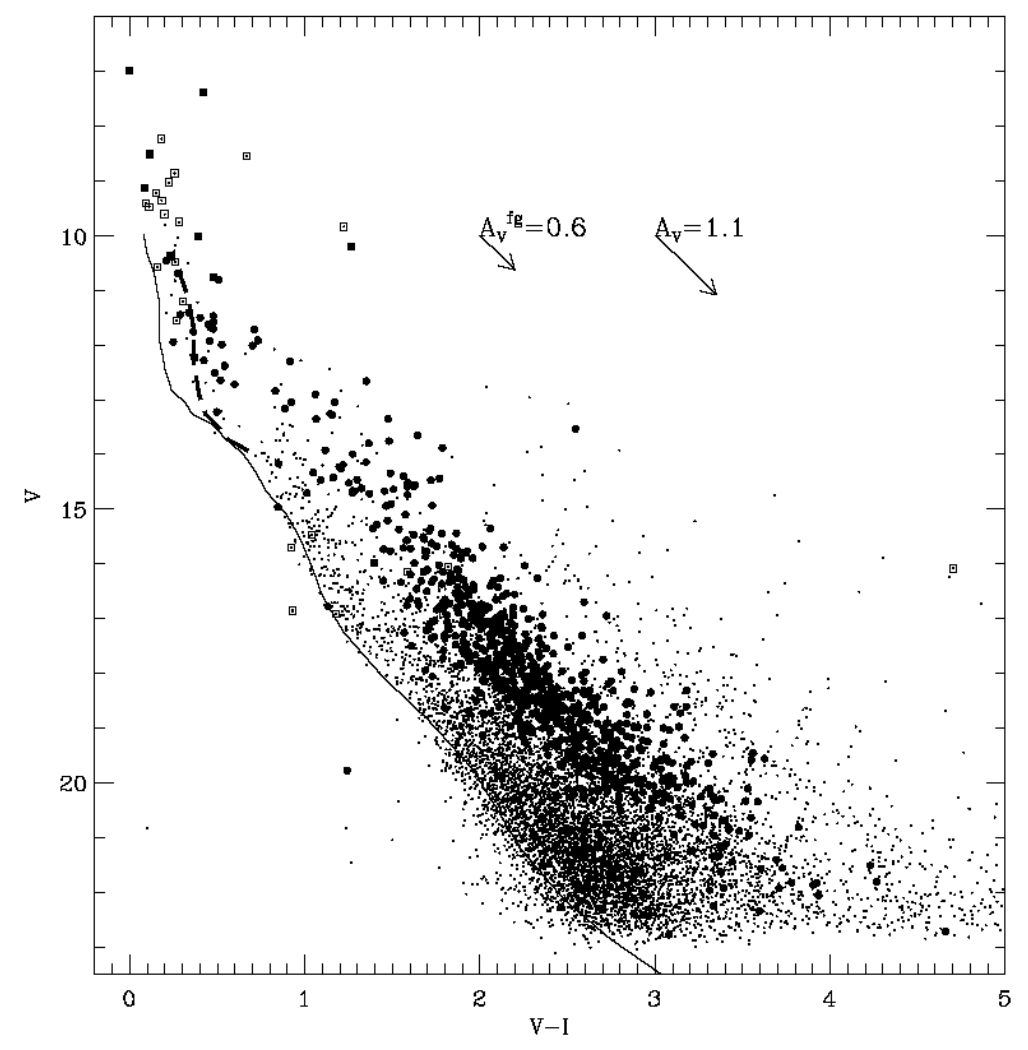

Fig. 3. $V$ vs. $V-I$ CMD of stars within the Chandra ACIS FOV. Dots indicate all stars in the optical catalog falling in the ACIS FOV, while large filled symbols indicate optical stars having an X-ray counterpart; stars added from the Sung et al. (2000) catalog are marked by squares. Lines and extinction vectors are as in Fig. 2.

sources, at magnitudes brighter than $V=17$, has already been analyzed in Damiani et al. (2004), using the photometric catalog of Sung et al. (2000). With the present survey it is now possible to identify the fainter cluster pre-main sequence region. In fact, most of the stars identified with an X-ray source are located within a well defined region, whose width may be due to an age spread and/or to binary stars. Only a small percentage of stars with an X-ray counterpart has photometry that is not consistent with that of the cluster. The field star contamination will be quantified in Sect. 6, but, at least qualitatively, it appears consistent with the percentage of contaminating X-ray sources ( $\$ 10 \%)$ given by Damiani et al. (2004).

As will be shown in Sect. 6, the cluster pre-main sequence region is comprised between the isochrones of 0.3 and $10 \mathrm{Myr}$; we used these isochrones to select possible cluster members from our optical catalog.

\section{Infrared data}

As discussed in the previous section, background stars are heavily obscured by the molecular cloud and therefore they appear at magnitudes much fainter than their intrinsic values. Nevertheless, optical diagrams presented in the previous section do not allow us to distinguish very faint objects with no or very low reddening from intrinsically bright background objects appearing as very faint stars due to their high reddening. Since near-infrared bands are very sensitive to reddened stars, we constructed the near-infrared CMDs using the $J H K_{\mathrm{S}}$ magnitudes from the 2MASS public catalog, with the aim of better characterizing the stellar populations in the WFI FOV. The diagram $H$ vs. $H-K$ of stars of the 2MASS catalog falling in the WFI FOV is shown in Fig. 4. We note that only stars with magnitudes measured from point spread-function fitting or aperture photometry were selected from the 2MASS catalog.

The large reddening effect can be quantified by inspecting the $H$ vs. $H-K$ diagram. In fact, in this diagram most stars are intrinsically colorless $(0 \lesssim H-K \lesssim 0.5$, Alves et al. 1998) and therefore the apparent $H-K$ color of a star is, to first order, a qualitative measure of the amount of extinction toward this star. This means that, apart from relatively few stars having reddening of the order of the average cluster reddening $E(B-V) \simeq 0.35$, corresponding to $E(H-K) \simeq 0.07$, the bulk of the stars has colors $H-K \gtrsim 0.3$, which, using the Mathis (1990) reddening law, corresponds to $A_{\mathrm{V}} \gtrsim 4.4$.

In this diagram we superposed three theoretical curves, viz. the ZAMS, the 0.3 and 10.0 Myr isochrones of Siess et al. (2000) (see Sect. 6), reddened using the cluster reddening value $E(B-V)=0.35$ and the Mathis (1990) reddening law. The location of these curves can be used to identify cluster stars and foreground field stars, affected by a reddening equal to or smaller than the average cluster reddening, from the bulk of the highly reddened stars $(H-K \gtrsim 0.3)$ that are either stars beyond the molecular cloud or cluster members showing IR excesses, i.e. classical T Tauri stars. 


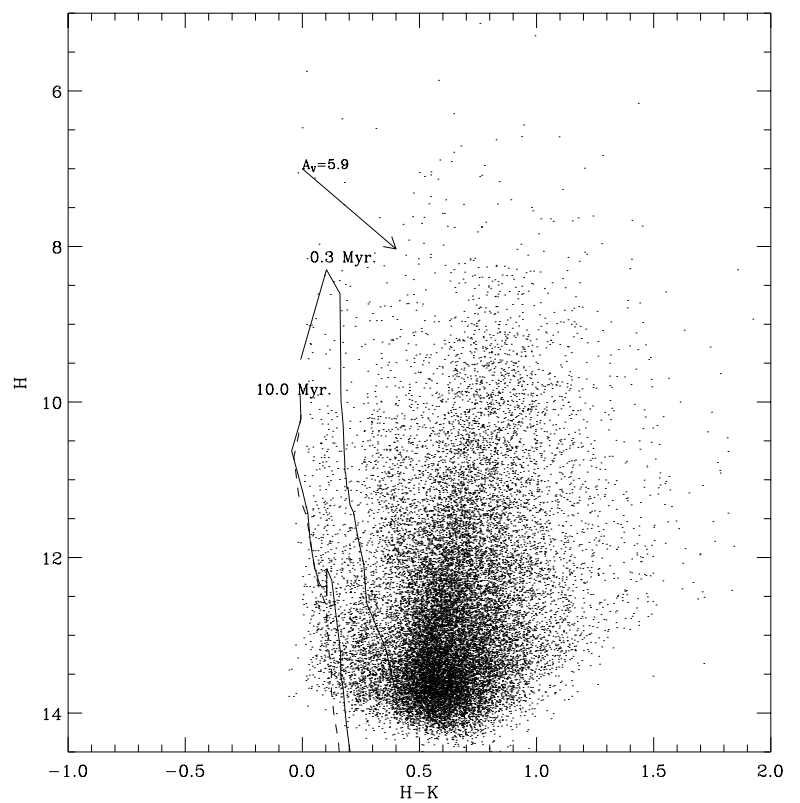

Fig. 4. $H$ vs. $H-K \mathrm{CMD}$ of all $2 \mathrm{MASS}$ point sources falling in the WFI FOV. The solid lines are the isochrones of 0.3 and $10.0 \mathrm{Myr}$, whereas the dashed line is the ZAMS at the cluster distance $d=$ $1250 \mathrm{pc}$.

\subsection{Optical and 2MASS detections}

In order to better characterize the stellar population of the optical catalog, we matched optical data with the 2MASS catalog, using a matching radius of 0.8 . We found systematic offsets in both right ascension and declination of $-0 .{ }^{\prime} 3$ and 0.16 , respectively; after correcting the optical catalog for this offset, a total of 15282 common stars were found.

Figures 5 and 6 are the color-magnitude and the color-color diagrams of possible cluster stars detected both in the optical and in the near-infrared catalogs. They are the 9613 stars (dots) that, in the $(V-I)$ vs. $V$ diagram, are found between the isochrones at 0.3 and $10.0 \mathrm{Myr}$, which is the estimated age spread of the cluster (see Sect. 6). Those stars for which an X-ray counterpart was also found are indicated as black bullets and, as asserted in Damiani et al. (2004), more than $90 \%$ of them are cluster members. In these diagrams, we also plotted the isochrones of 0.3 and $10 \mathrm{Myr}$, indicated by the solid lines, and the reddening vector. The dotted line in Fig. 6 corresponds to the locus of classical T Tauri stars (Meyer et al. 1997).

If we compare Figs. 4 and 5 we note that most of the reddened stars (e.g. $H-K \gtrsim 0.6$ ) in the IR catalog are not seen in the optical bands, at least down to the limiting magnitude of this survey. Most of the stars detected both in the optical and in the near-infrared bands have $H-K \lesssim 0.6$, but there are objects with $(H-K)$ up to 1.5 . Using the extinction law of Mathis (1990), these values indicate that most of the stars in the optical catalog have extinction $A_{\mathrm{V}} \lesssim 8.8 \mathrm{mag}$, while the high-reddening objects can have $A_{\mathrm{V}}$ up to $20 \mathrm{mag}$.

Figure 5 clearly shows that many cluster members (black bullets) are located near the young theoretical isochrone,

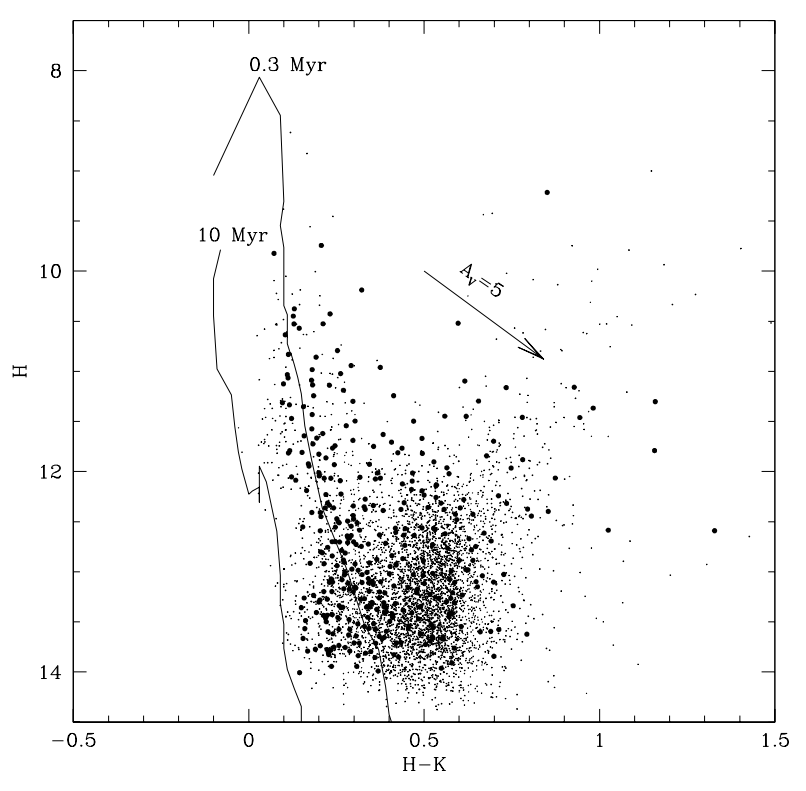

Fig. 5. $H$ vs. $H-K$ CMD of the stars detected both in the optical and in the near-infrared catalogs. Dots are stars with ages between 0.3 and 10 million years as selected in the $(V-I)$ vs. $V$ diagram while black bullets are those with an X-ray counterpart. The solid lines are the isochrones of 0.3 and $10.0 \mathrm{Myr}$.

but a significant number is also found redwards, with $(H-K)$ up to 1.3. Therefore, whereas the cluster members (selected for their X-ray emission) are located in a well defined region of the optical CMD, they are much more "dispersed" in the $H$ vs. $H-K$ diagram. This is probably due to the fact that a large fraction of cluster members shows significant excesses in the IR colors.

This can be seen also in the $H-K$ vs. $I-J$ diagram of Fig. 6 where many of the 2MASS-optical candidate members are found in a strip around the theoretical curves, while at low reddening a clump of stars is found at $H-K \gtrsim 0.4$ and $(I-J) \gtrsim 2.5$. As already discussed, the latter objects are the highest reddened stars and have $(V-I) \gtrsim 3.5$ in the $V$ vs. $(V-I)$ diagram. Among the 2MASS-optical sources that are also X-ray detected, less than $10 \%$ are high-reddening sources $(I-J \gtrsim 2.5)$. Many of the X-ray detected cluster members are instead found along the theoretical isochrones, but there is also a significant number of stars with large $H-K$, but in a different direction with respect to the reddening vector. This supports the hypothesis that these X-ray sources are classical T Tauri stars with IR excesses, as also suggested by Damiani et al. (2004).

These properties are also found in the other combined optical-IR color-magnitude and color-color diagrams and therefore we conclude that optical and 2MASS combined data alone cannot be used to select a clean sample of PMS cluster members.

\section{Masses, ages and spatial distribution}

Using the Sung et al. (2000) photometric catalog, Damiani et al. (2004) determined masses and ages of X-ray detected stars 


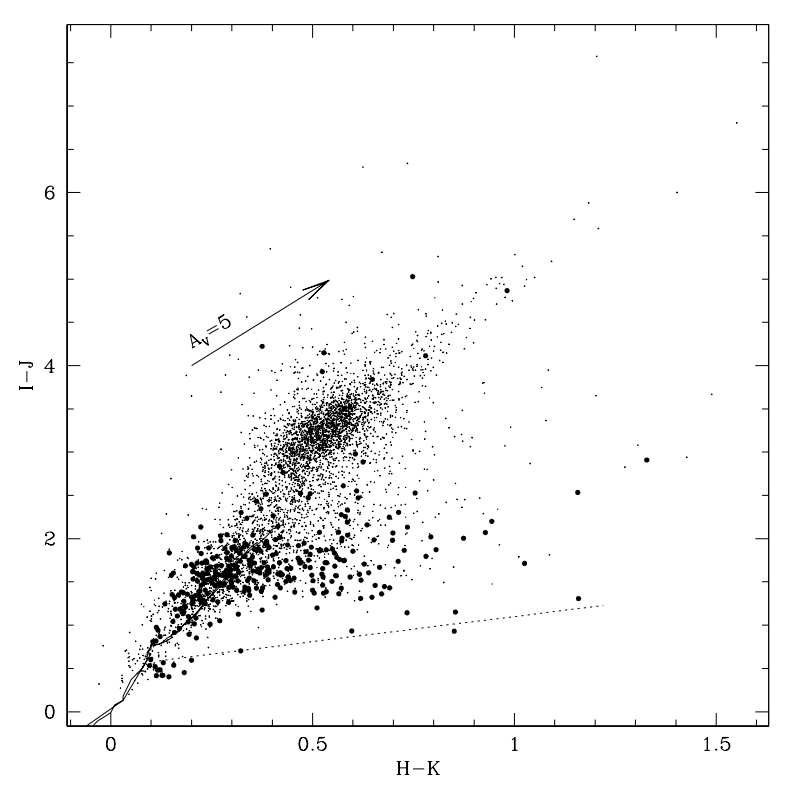

Fig. 6. $I-J$ vs. $H-K$ color-color diagram of the stars detected both in the optical and in the near-infrared catalogs. Symbols are as in Fig. 5. The solid lines are the isochrones of 0.3 and $10.0 \mathrm{Myr}$, whereas the dotted line corresponds to the locus of classical T Tauri stars (Meyer et al. 1997).

brighter than $V=17$, finding stars down to $0.5 M_{\odot}$, with completeness only for masses larger than $1.5 M_{\odot}$. In addition, they found that the median age of the cluster stars in the central region is $0.82 \mathrm{Myr}$ with a maximum spread of $4 \mathrm{Myr}$. These values were derived assuming a cluster distance larger than the value estimated in this work, which led those authors to overestimate luminosities and masses of the stars.

In the present work we have recomputed masses and ages of the probable cluster members, derived by interpolating the theoretical tracks and isochrones calculated by Siess et al. (2000) to the positions of the stars in the $V$ vs. $V-I$ CMD for which an X-ray counterpart has been found. Of these stars more than $90 \%$ are cluster members, although it is unknown which percentage they are of the whole cluster population. The completeness of this sample is discussed in Sect. 7.

We used the models of Siess et al. (2000) because it was shown that masses predicted with these tracks are consistent with masses estimated dynamically (Simon et al. 2000). In addition they are in good agreement with the pre-main sequence models of Baraffe et al. (1998), which are available only for ages older than $1 \mathrm{Myr}$. We are, however, aware of the large uncertainties of these models for ages younger than $1 \mathrm{Myr}$, which are based on oversimplified initial conditions, as discussed in Baraffe et al. (2002). Nevertheless, they are the most complete set of theoretical tracks available in the literature, and have already been used in recent studies of star formation regions (e.g. Flaccomio et al. 2003).

Since metallicity has never been estimated for NGC 6530 , we assume for this cluster a solar metallicity and we consider the Siess et al. (2000) models with $Z=0.02, Y=$ $0.277, X=0.703$ and no overshooting. To convert effective temperatures and luminosities of the adopted model to the empirical $V$ vs. $V-I$ plane, we used the conversion table of Kenyon \& Hartmann (1995) where both optical and infrared colors are available.

Figure 7 shows the $V$ vs. $V-I$ CMD of X-ray detected cluster members with superimposed the adopted theoretical curves. We note that the curves were reddened using the value $E(B-V)=0.35$ given by Sung et al. (2000) and in agreement with our data (see Sect. 3.1), $A_{\mathrm{V}}=3.1 \times E(B-V)$ and the reddening law $E(V-I)=1.3 \times E(B-V)$ (Munari \& Carraro 1996). The absolute magnitudes of the theoretical models were transformed to apparent magnitudes assuming the distance $d \simeq$ $1250 \mathrm{pc}$ derived in Sect. 3.1. Dotted lines are the evolutionary tracks for masses between 0.25 and $7 M_{\odot}$, while solid lines are the isochrones for ages between 0.1 and $100 \mathrm{Myr}$, the latter being very similar to the ZAMS (dashed line).

Due to the strong variation of the surface spatial density of this area, the cluster is expected to be affected by a nonnegligible differential reddening. Evidence of such effect was found by Sung et al. (2000). This implies that the age and mass estimates are affected by uncertainties due to differential reddening. However, as discussed in Damiani et al. (2004), for most of the low mass cluster member, the reddening vector direction is almost parallel to the isochrones and thus the age estimates are less affected than the mass estimates, which can have an uncertainty up to $0.5 M_{\odot}$. Nevertheless, this uncertainty does not drastically affect the determination of the Initial Mass Function since it is smaller than the considered mass bins. Figure 7 shows that most of the X-ray detected stars are included between the theoretical isochrones of 0.3 and $10 \mathrm{Myr}$. We expect that stars outside this age range are mainly contaminating objects or highly reddened cluster members. We note that the observed spread (1.5-2 mag) is larger than that expected from binarity or photometric uncertainty since only data with errors smaller than $0.2 \mathrm{mag}$ have been considered. The age distribution for the stars in this age range is shown in Fig. 8; it is quite symmetric and concentrated, with a median age of $2.3 \mathrm{Myr}$.

Because of the position of the cluster with respect to the Hourglass nebula, a sequential process of the star formation was proposed for this cluster (Lada et al. 1976). In particular, Damiani et al. (2004) suggested that the star formation has progressed from north to south. In order to find further evidence for this effect, we plotted in Fig. 9 the spatial distributions of the optical/X-ray detected sources in four different age ranges. Our results support the conclusion of Damiani et al. (2004) since very young stars are present (almost) only in the southern cluster region, while the spatial distribution becomes more uniform for higher ages. To test the validity of this conclusion, we have made a statistical analysis using the age distributions in 5 different spatial regions, namely the cluster center included in a square region of sides 7.2 arcmin, as defined in Damiani et al. (2004), and 4 outer regions (see Fig. 9). The cumulative age distributions in Fig. 10 show a clear age difference between the southern and central regions and the northern ones. Using the two sample Kolmogorov-Smirnov tests, we find that the cumulative distribution functions of each of the southern and central 


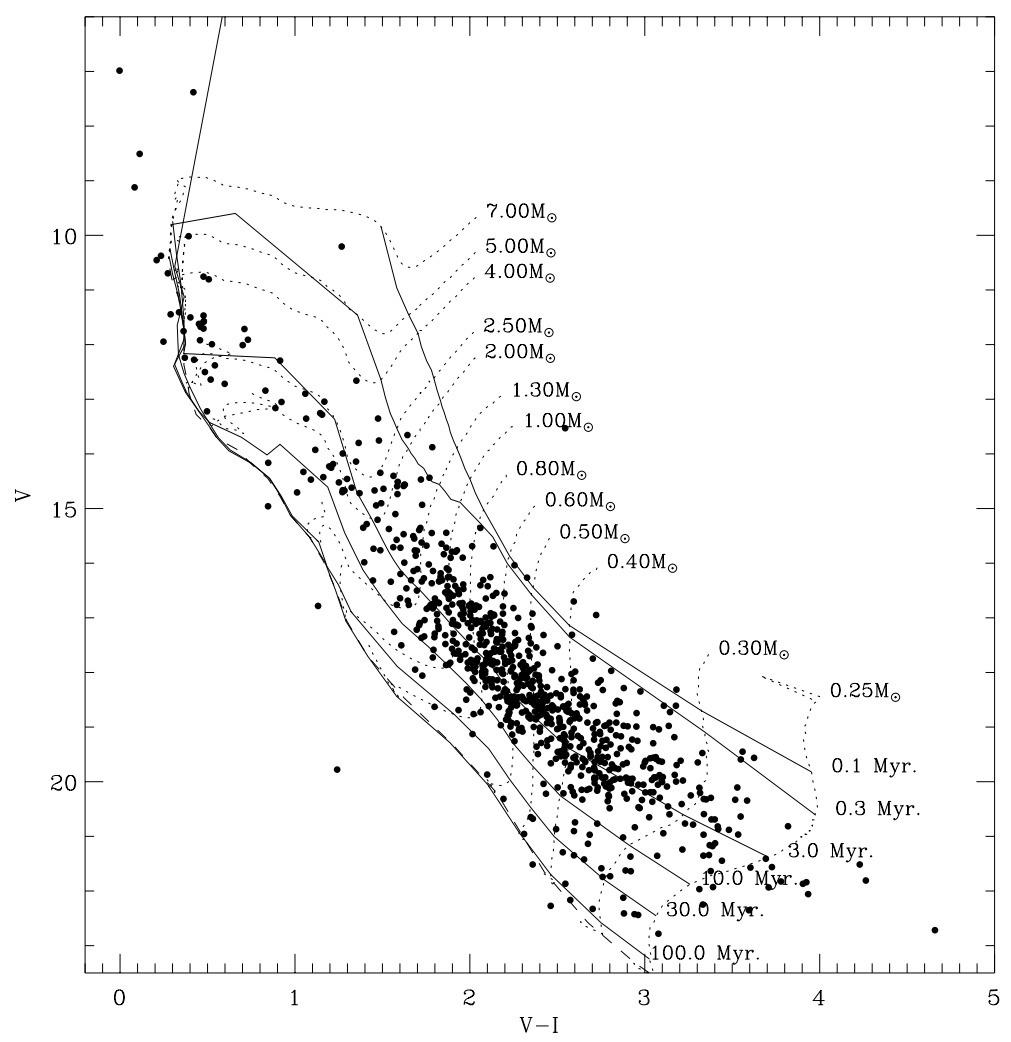

Fig. 7. $V$ vs. $V-I$ CMD of the stars within the Chandra ACIS FOV with an X-ray counterpart. The dotted lines are the solar metallicity evolutionary tracks computed by Siess et al. (2000); the corresponding mass value in solar mass units is given at the red end of each track. The solid lines are the corresponding isochrones for ages between 0.1 and $100 \mathrm{Myr}$, while the dashed line is the ZAMS at the distance of the cluster. We note that the theoretical curves were reddened using the cluster reddening value given by Sung et al. (2000) and the reddening law of Munari \& Carraro (1996).

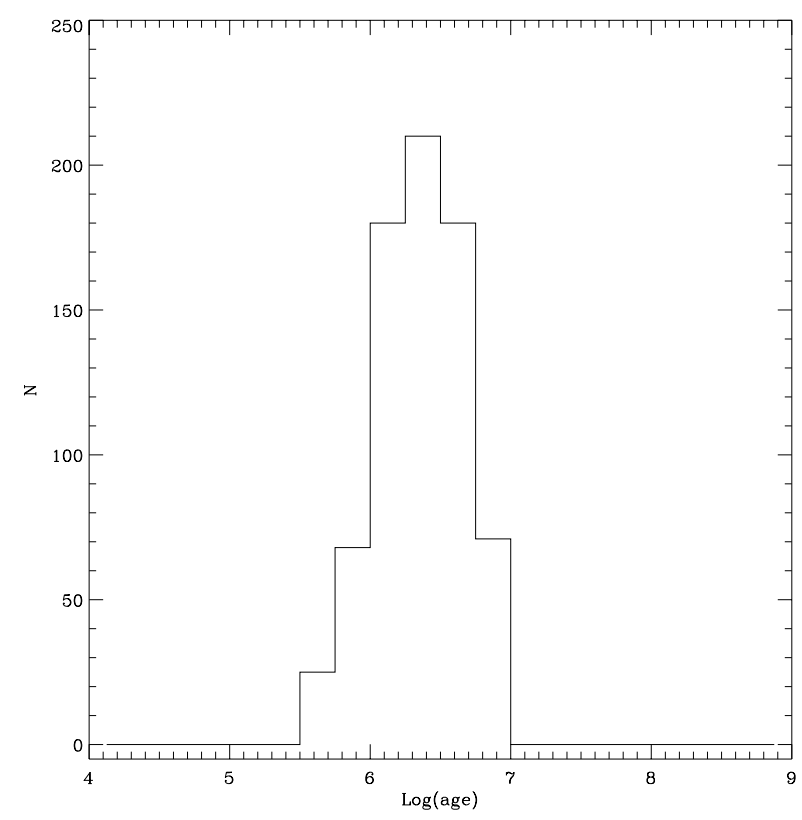

Fig. 8. Age distribution of all optical/X-ray detected sources; the star age was estimated by comparing the star positions in the $V$ vs. $V-I$ CMD with the isochrones computed by Siess et al. (2000). The median age value is $2.3 \mathrm{Myr}$. regions are different from the northern regions at a significance level greater than $99 \%$.

Figure 11a shows the spatial distribution of all the 8975 optical sources in the Chandra ACIS FOV to which no photometric selection was applied. This distribution is clearly dominated by field stars and does not show any evidence of central clustering. The very strong absorption of the nebula causes a very patchy spatial distribution of stars. Figure 7 shows that most of the optical/X-ray sources have ages in the range $(0.3-10.0)$ Myr. This means that stars with ages outside this range are not photometrically cluster members. Therefore, we selected all optical stars with ages in this range and we defined them as "optical candidate cluster members". The spatial distribution of these stars is shown in Fig. 11b. Although it is still dominated by field stars, some evidence of star clustering is visible.

From this sample, we defined as "optical/X-ray cluster members" all the 737 optical sources with an X-ray counterpart and age consistent with that of the cluster. The spatial distribution of these stars is shown in Fig. 11c, where strong evidence of clustering is seen. The clustered distribution of these stars allows us to confirm the conclusion that most of the optical/X-ray detected stars are cluster members, and clearly shows how efficient X-ray observations are for selecting cluster members. We note that these latter stars are about $90 \%$ of the whole sample 

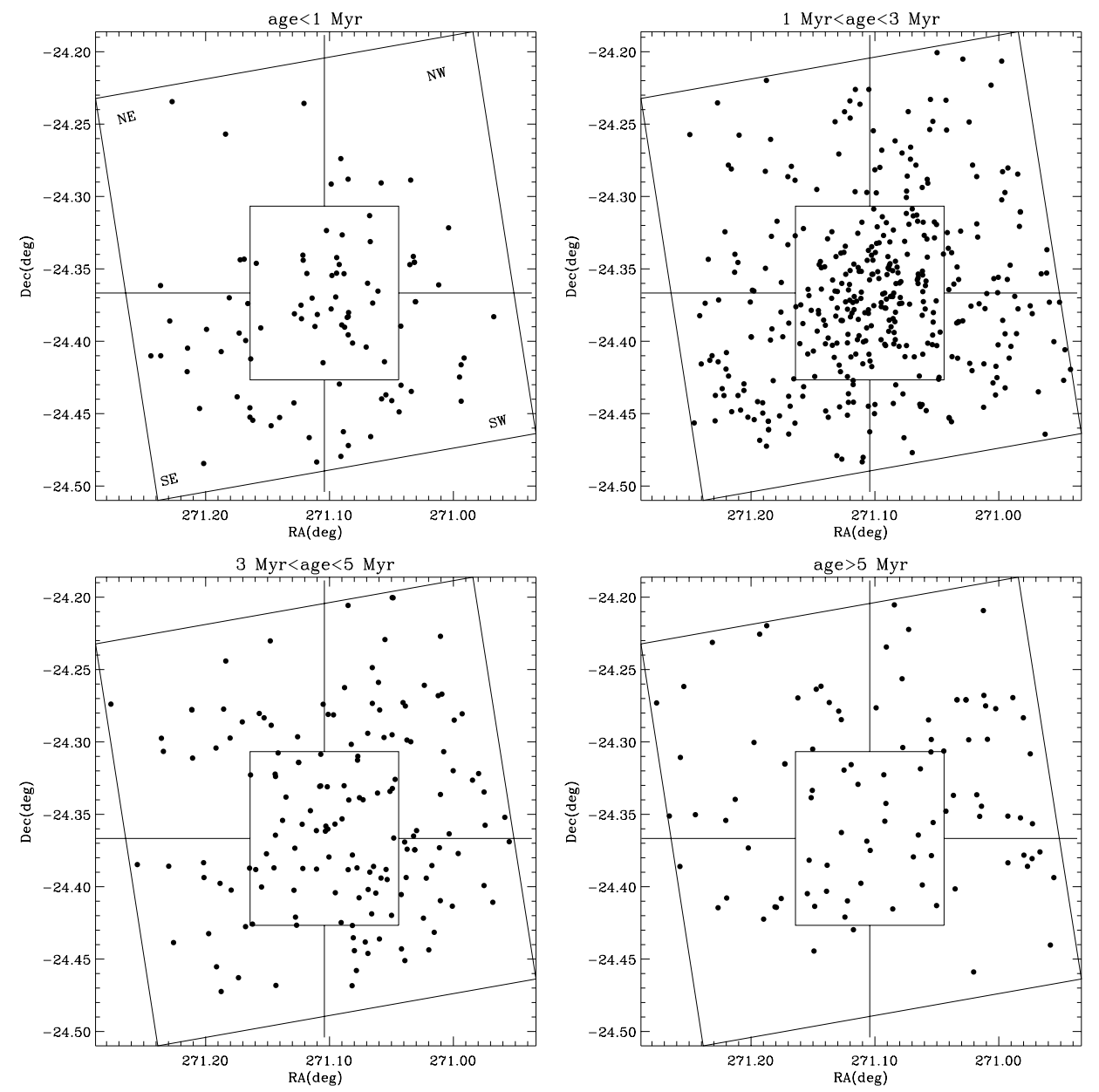

Fig. 9. Spatial distributions of the optical/X-ray detected stars in the four age ranges.

of optical/X-ray sources (828 stars), in agreement with the field star contamination estimated in Damiani et al. (2004).

Figure 11d shows the spatial distribution of the optical candidate cluster sample from which the 737 optical/X-ray cluster members have been subtracted. These stars do not show any evidence of clustering; on the contrary they show the same patchy pattern of the contaminated sample and this suggests that most of them are field stars. We conclude that our optical/X-ray sample suffers from little contamination, and includes a very large fraction of the cluster members within the studied magnitude limit.

\section{Luminosity and initial mass functions}

Figure 12 shows the $V$ magnitude distribution of the optical/X-ray cluster members (solid histogram) and that of all optical candidate cluster members (dashed histogram) defined in Sect. 6. These distributions can be considered only as lower and upper limits to the Luminosity Function of the cluster. In fact, because of the limited sensitivity of the Chandra ACIS detector, we expect that the sample of "optical/X-ray cluster members" is not complete but, as we have discussed above, the expected incompleteness should be small although the sample includes only about $21 \%$ of the "optical candidate cluster members". Because of the strong gradient of the spatial

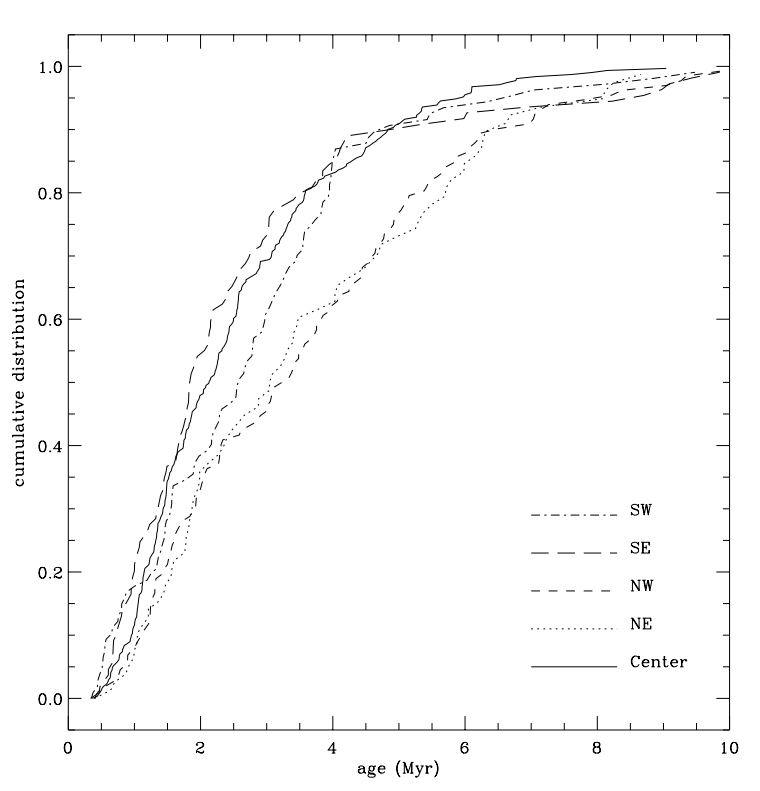

Fig. 10. Age distributions of the optical/X-ray sources in the five distinct spatial subregions defined in Fig. 9.

density distribution of the whole region it is impossible to choose a suitable region of the FOV to be used as "control 

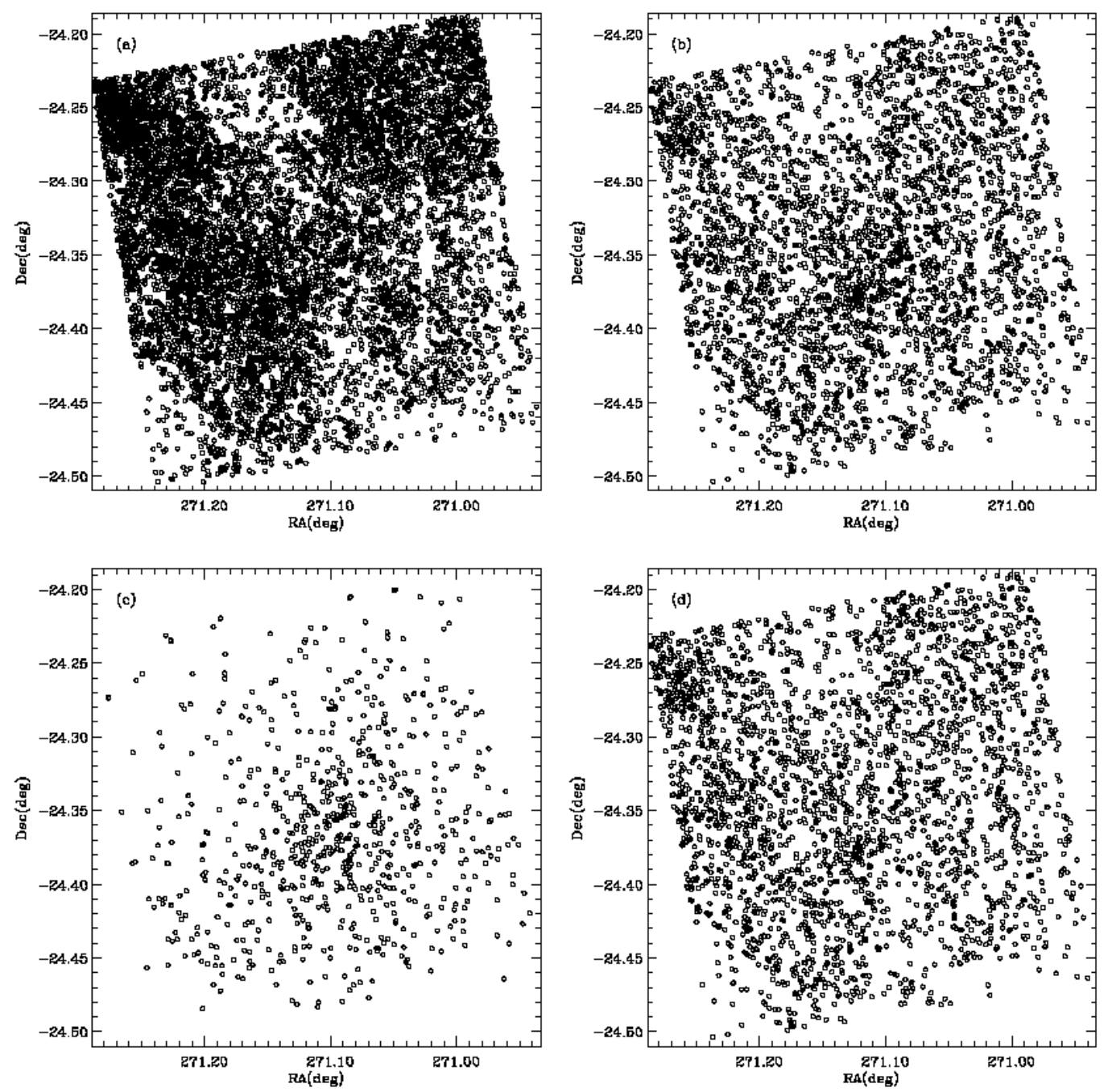

Fig. 11. a) Spatial distribution of all the 8975 optical sources within the Chandra ACIS FOV; b) same distribution for the 3427 stars with magnitudes and colors consistent with that of the cluster, whose age is between 0.3 and $10.0 \mathrm{Myr}$ (referred to as "optical candidate members"); c) spatial distribution of the $737 \mathrm{Optical} / \mathrm{X}$-ray cluster members; d) spatial distribution of the 2690 optical candidate member sample after subtracting the 737 optical/X-ray cluster members.

field" to estimate the field star contamination of the optical candidate cluster members.

In addition, in the case of pre-main sequence stars, the observed luminosity function cannot be directly converted into mass function. For these reasons, we have constructed the mass function using the "incomplete" sample of "optical/X-ray cluster members", that includes stars with ages derived from the CMD between 0.3 and 10 Myr. For these stars, we directly estimated the stellar masses from their position on the $V$ vs. $V-I$ CMD, as described in Sect. 6. In order to obtain the mass function of the whole cluster, we first considered the completeness of the optical catalog, using the results of the artificial star tests described in Sect. 3.1. To obtain the optical complete mass distribution, the fractions of the retrieved artificial stars in each $V$ and $I$ magnitude bin were interpolated to the position of the stars in the $V$ vs. $V-I$ CMD. The minimum fraction computed in each mass bin is reported in Col. 2 of Table 6.

To take into account the X-ray incompleteness of member selection, we corrected the mass function assuming that the fraction of NGC 6530 members detected as X-ray sources is, at the same sensitivity, equal to that detected for a cluster of similar age for stars of a given mass.

The most suitable region for this comparison is certainly the Orion Nebula Cluster, one of the best studied clusters, for which all cluster members are known from extensive optical and infrared surveys and for which Chandra X-ray observations were recently published by Flaccomio et al. (2003) and Feigelson et al. (2002). Figure 3 of Flaccomio et al. (2003) shows the cumulative X-ray Luminosity Functions relative to the complete optical sample of stars in the Orion Nebula Cluster, for eight ranges of mass from 0.1 to $50 M_{\odot}$. In order to obtain an appropriate correction to the NGC 6530 Mass Function derived from the optical/X-ray star sample, we considered that the sensitivity of the NGC 6530 Chandra ACIS observation allows us to measure X-ray luminosities greater than $\log \left(L_{\mathrm{X}}\right) \simeq 29.2(\mathrm{erg} / \mathrm{s})$ in the central region and greater than $\log \left(L_{\mathrm{X}}\right) \simeq 29.7(\mathrm{erg} / \mathrm{s})$ in the external region of the ACIS FOV for an X-ray spectrum with $k T=1 \mathrm{keV}$, and $N_{\mathrm{H}}$ 
Table 6. Mass Function of NGC 6530.

\begin{tabular}{cccccc}
\hline \hline$\Delta M$ & Min. optical & $\begin{array}{c}\text { X-ray fraction for } \\
\left(M_{\odot}\right)\end{array}$ & $\begin{array}{c}\left(\frac{\Delta N}{\Delta \log M}\right)_{\min } \\
\text { lcompleteness }\end{array}$ & $\begin{array}{c}\left(\frac{\Delta N}{\Delta \log M}\right) \\
\log \left(L_{\mathrm{X}}\right)=29.7 \log \left(L_{\mathrm{X}}\right)=29.2\end{array}$ & $\left.\begin{array}{c}\Delta N \\
\Delta \log M\end{array}\right)_{\max }$ \\
\hline $0.16-0.20$ & 0.1 & 0.10 .5 & 184 & 549 & 914 \\
$0.20-0.25$ & 0.4 & 0.10 .5 & 417 & 1245 & 2073 \\
$0.25-0.38$ & 0.9 & 0.50 .7 & 1189 & 1500 & 1812 \\
$0.38-0.50$ & 0.9 & 0.50 .7 & 2082 & 2626 & 3171 \\
$0.50-0.75$ & 0.9 & 0.70 .9 & 1118 & 1272 & 1425 \\
$0.75-1.00$ & 1.0 & 0.70 .9 & 730 & 831 & 931 \\
$1.00-1.50$ & 1.0 & 0.81 .0 & 416 & 450 & 483 \\
$1.50-2.00$ & 1.0 & 0.81 .0 & 215 & 233 & 250 \\
$2.00-2.50$ & 1.0 & 0.80 .8 & 274 & 274 & 274 \\
$2.50-3.00$ & 1.0 & 0.80 .8 & 227 & 227 & 227 \\
$3.00-6.50$ & 1.0 & 0.50 .5 & 28 & 28 & 28 \\
\hline
\end{tabular}

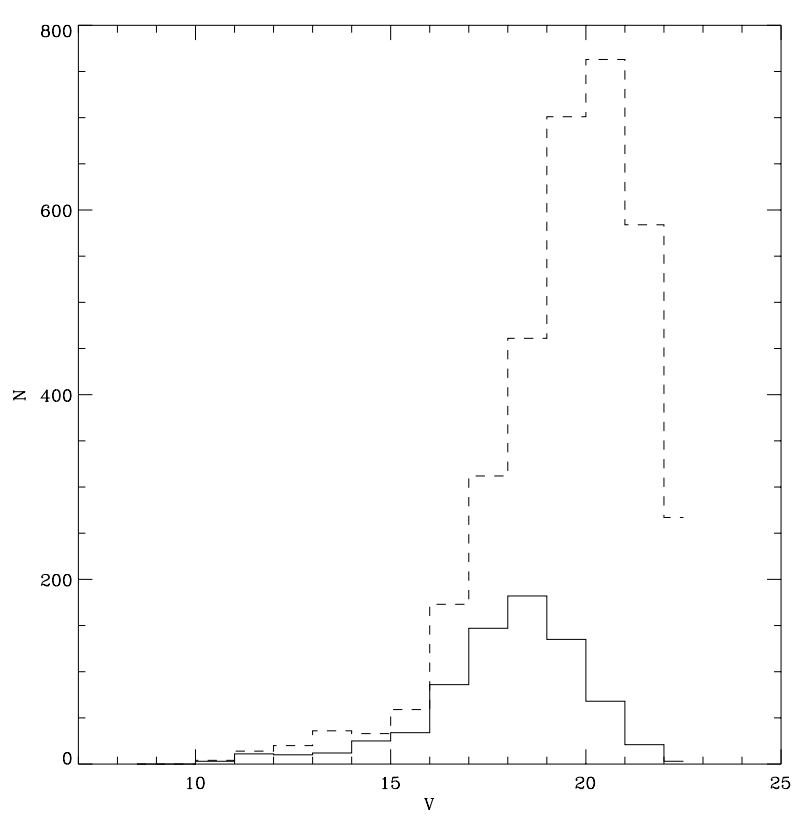

Fig. 12. $V$ magnitude distribution of the X-ray cluster members (solid histogram) and of all optical candidate cluster members (dashed histogram).

corresponding to the optical extinction (Damiani et al., in preparation). For each mass range considered by Flaccomio et al. (2003), we took the fraction of X-ray detected stars above $\log \left(L_{X}\right)=29.2(\mathrm{erg} / \mathrm{s})$ and $\log \left(L_{X}\right)=29.7(\mathrm{erg} / \mathrm{s})$, given in Cols. 3 and 4 of Table 6 , and we used these fractions to obtain the minimum and maximum correction to our incomplete Mass Function. The mass function of NGC $6530 \Delta N / \Delta \log M$ is therefore taken as the average of the minimum and maximum distributions $(\Delta N / \Delta \log M)_{\min }$ and $(\Delta N / \Delta \log M)_{\max }$, resulting from these corrections.

The results are given in Table 6 and shown in Fig. 13 where the dashed histogram shows the X-ray incomplete mass function derived from the X-ray cluster members, while the black

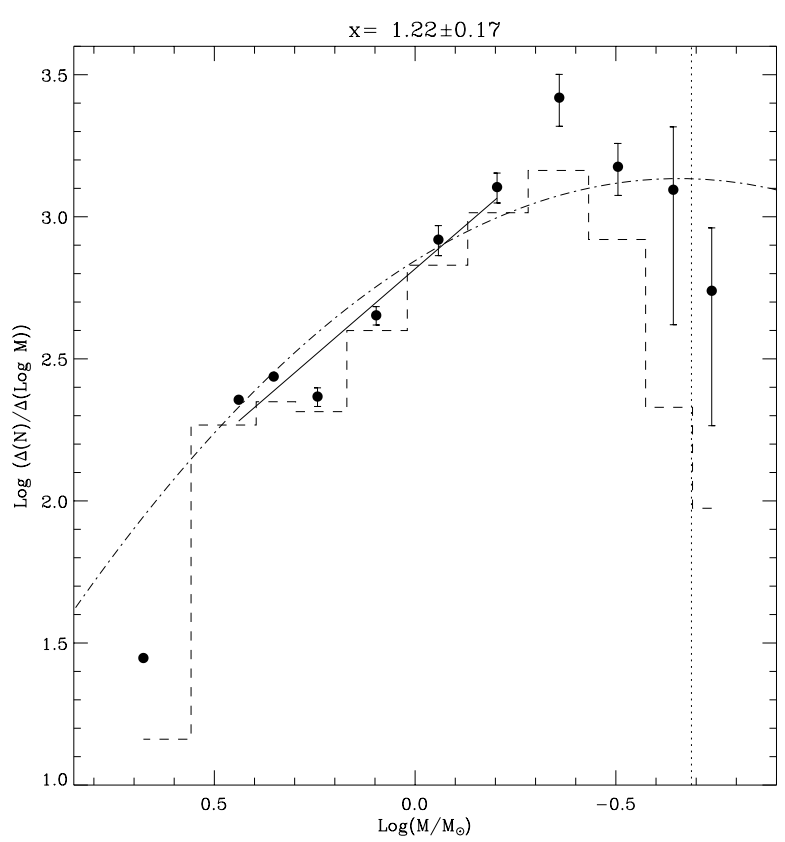

Fig. 13. The dashed histogram shows the $\mathrm{X}$-ray incomplete mass function derived from the X-ray cluster members, while the black bullets indicate the mass function of the cluster corrected according to the procedure described in Sect. 7 (average values between the minimum and maximum corrections, indicated in the figure by the error bars). The field star mass function given in Chabrier (2003), arbitrarily normalized to the present data, is plotted as the dotted-dashed line. The solid line is the power law fitting the NGC 6530 mass function in the range (0.6-4.0) $M_{\odot}$. The power law index and the corresponding rms uncertainty are also indicated. The vertical dotted line corresponds to the value at which the photometric completeness limit is larger than $40 \%$.

bullets indicate the corrected mass function of the cluster; the error bars show the minimum and maximum corrected distributions. Note that, with the exception of the lowest mass bin, the optical incompleteness is always smaller than $60 \%$ and it makes sense to correct it as described before. 
Since the median age of the cluster is smaller than the typical time scale for dynamical evolution $(\sim 100 \mathrm{Myr})$ and/or stellar evolution, the measured mass function is equal to the Initial Mass Function. The cluster IMF increases if one goes down from 6.5 to about $0.4 M_{\odot}$, where it shows a peak, and decreases for lower masses. In the mass range (0.6-4.0) $M_{\odot}$, it was fitted with a power law of index $x=1.22 \pm 0.17$, which is consistent with the Salpeter index 1.35. The lower limit of the mass range was chosen equal to that used by Muench et al. (2002b) in the Orion Nebula cluster, in order to compare the slope of the IMF of these similar clusters in the same mass range. The power law index obtained for NGC 6530 is also consistent with the index obtained for the coeval Trapezium, Taurus and IC 348 clusters in the same mass range (Muench et al. 2002a,b; Briceño et al. 2002; Muench et al. 2003). In the common mass range, while the IMF of most star forming regions and open clusters, such as Trapezium, IC 348, Lambda Orionis (Navascues et al. 2004), and the Pleiades (Bouvier et al. 1998) flattens at about 0.8 $M_{\odot}$, the NGC 6530 IMF appears to decrease for masses lower than about $0.4 M_{\odot}$. A quantitative comparison with the IMF of other star forming regions or open clusters for very low masses cannot be performed with the available data, since the NGC 6530 IMF is limited to $M \geq 0.2 M_{\odot}$.

The NGC 6530 IMF has also been compared with the field star IMF derived by Chabrier (2003) that is displayed in Fig. 13 by the dotted-dashed line. The NGC 6530 IMF is reminiscent of the lognormal shape derived for the galactic disk field stars.

The total mass of the optical/X-ray members is about $560 M_{\odot}$; using the corrections for optical and X-ray incompleteness we find that the total mass of the cluster, including stars down to $0.4 M_{\odot}$, is between 700 and $930 M_{\odot}$.

\section{Summary and conclusions}

We used $B V I$ images taken with the WFI camera of the $\mathrm{ESO} / 2.2 \mathrm{~m}$, available at the ESO/ST-ECF Science Archive, to obtain multi-band photometry of the very young open cluster NGC 6530. This cluster is located in a star formation region known as the Lagoon Nebula (M 8), which is one of the brightest nebulae in the solar vicinity. The whole field shows evidence of strong obscuration due to the dense molecular cloud located just behind the cluster.

The present photometric catalog reaches down to $V \simeq 23$ and allows us to significantly increase the knowledge of this cluster. In fact, the most recent photometric survey of this cluster was published by Sung et al. (2000) and reaches down to only $V=17$. From their survey Sung et al. (2000) derived a cluster distance of $1800 \mathrm{pc}$ corresponding to a distance modulus of $\left(V-M_{\mathrm{V}}\right)_{0}=11.25$, which is much larger than the value $d=1250 \mathrm{pc}$, inferred from the present work by considering a more complete CMD and taking advantage of the total absence of background stars beyond the main sequence locus at the cluster distance.

The near-infrared CMDs of the same FOV, obtained using $J H K$ magnitudes taken from the 2MASS catalog, suggest that this region includes a large number of very reddened objects that are not present in the optical CMDs, at least within our limiting magnitude. A few of them have been detected as X-ray sources.

Optical and near-infrared data alone do not allow us to identify the cluster stars in the CMDs, since they do not form a well defined sequence but, on the contrary, they populate a wide region in the CMDs. For this reason it has been crucial to cross-correlate optical data with X-ray sources detected with the Chandra ACIS instrument and very recently published by Damiani et al. (2004). A total of 828 optical/X-ray sources was found and placed in the CMD. $90 \%$ of these stars are very probable cluster members and identify a very well defined pre-main sequence region, that is the cluster locus comprised between the 0.3 and $10 \mathrm{Myr}$ isochrones.

Masses and ages of these cluster members were estimated using the evolutionary tracks computed by Siess et al. (2000). The age distribution of these stars indicates that the median age of the cluster is about $2.3 \mathrm{Myr}$. A statistically significant trend in the spatial distribution of these stars was found as a function of age, as already suggested by Lada et al. (1976) and Damiani et al. (2004). This suggests a sequential star formation process from north to south.

The Initial Mass Function derived from the photometrically selected stars with an X-ray counterpart was corrected for incompleteness of the $\mathrm{X}$-ray data assuming that the fraction of NGC 6530 members detected as X-ray sources is, at each given sensitivity, the same as that detected in the Orion Nebula Cluster. In the mass range $(0.6-4.0) M_{\odot}$, the corrected Initial Mass Function can be represented by a power law with index $x=1.22 \pm 0.17$, consistent with the Salpeter index 1.35, while at smaller masses it shows a peak and then it starts to decrease. The resulting IMF is similar to that obtained for coeval clusters, such as Trapezium, Taurus and IC348, in the same mass range.

Acknowledgements. This work is part of the Ph.D. Thesis of L.P.; we acknowledge financial support from the Italian MIUR, and E. Flaccomio for useful suggestions that greatly improved our analysis. We thank an anonymous referee for useful comments and suggestions.

\section{References}

Alcala, J. M., Krautter, J., Schmitt, J. H. M. M., et al. 1995, A\&AS, 114,109

Alves, J., Lada, C. J., Lada, E. A., et al. 1998, ApJ, 506, 292

Baraffe, I., Chabrier, G., Allard, F., et al. 1998, A\&A, 337, 403

Baraffe, I., Chabrier, G., Allard, F., et al. 2002, A\&A, 382, 563

Bouvier, J., Stauffer, J. R., Martin, E. L., et al. 1998, A\&A, 336, 490

Briceño, C., Luhman, K. L., Hartmann, L., et al. 2002, ApJ, 580, 317

Chabrier, G. 2003, ApJ, 586, L133

Chini, R., \& Neckel, T. 1981, A\&A, 102, 171

Cutri, R. M., et al. 2003, 2MASS All-Sky Catalog of Point Sources, University of Massachusetts and Infrared Processing and Analysis Center, IPAC/CAlifornia Institute of Technology

Damiani, F., Flaccomio, E., Micela, G., et al. 2004, ApJ, 608, 781

Feigelson, E. D., Broos, P., Gaffney, J. A., et al. 2002, ApJ, 574, 258

Flaccomio, E., Micela, G., Sciortino, S., et al. 2000, A\&A, 355, 651

Flaccomio, E., Damiani, F., Micela, G., et al. 2003, ApJ, 582, 382

Hiltner, W. A., Morgan, W. W., \& Neff, J. S. 1965, ApJ, 141, 183

Kenyon, S. J., \& Hartmann, L. 1995, ApJS, 101, 117

Kilambi, G. C. 1977, MNRAS, 178, 423 
Lada, C. J., Gottlieb, C. A., Gottlieb, E. W., et al. 1976, ApJ, 203, 159 Landolt, A. U. 1992, AJ, 104, 340

Loktin, A., Zakharova, P., Gerasimenko, T., et al. 1997, Baltic Astronomy, 6, 316

Loktin, A. V., \& Beshenov, G. V. 2001, Astron. Lett., 27, 386

Mathis, J. S. 1990, ARA\&A, 28, 37

McCall, M. L., Richer, M. G., \& Visvanathan, N. 1990, ApJ, 357, 502

Meyer, M. R., Calvet, N., \& Hillenbrand, L. 1997, AJ, 114, 288

Momany, Y., Vandame, B., Zaggia, S., et al. 2001, A\&A, 379, 436

Muench, A. A., Lada, E. A., \& Lada, C. J. 2002a, BAAS, 34, 1210

Muench, A. A., Lada, E. A., Lada, C. J., et al. 2002b, ApJ, 573, 366

Muench, A. A., Lada, E. A., Lada, C. J., et al. 2003, AJ, 125, 2029

Munari, U., \& Carraro, G. 1996, A\&A, 314, 108

Navascues, D. B. Y., Stauffer, J. R., Bouvier, J., et al. 2004, ApJ, 610, 1064

Randich, S., Schmitt, J. H. M. M., Prosser, C. F., et al. 1995, A\&A, 300, 134

Rauw, G., Nazé, Y., Gosset, E., et al. 2002, A\&A, 395, 499
Sagar, R., \& Joshi, U. C. 1978, MNRAS, 184, 467

Siess, L., Dufour, E., \& Forestini, M. 2000, A\&A, 358, 593

Simon, M., Dutrey, A., \& Guilloteau, S. 2000, ApJ, 545, 1034

Sterzik, M. F., Alcala, J. M., Neuhaeuser, R., et al. 1995, A\&A, 297, 418

Stetson, P. B. 1987, PASP, 99, 191

Stetson, P. B. 1990, PASP, 102, 932

Stetson, P. B. 1994, PASP, 106, 250

Stetson, P. B. 2000, PASP, 112, 925

Sung, H., \& Bessell, M. S. 1999, MNRAS, 306, 361

Sung, H., Chun, M., \& Bessell, M. S. 2000, AJ, 120, 333

The, P. 1960, ApJ, 132, 40

van Altena, W. F., \& Jones, B. F. 1972, A\&A, 20, 425

van den Ancker, M. E., The, P. S., Feinstein, A., et al. 1997, A\&AS, 123, 63

Walker, M. F. 1957, ApJ, 125, 636

Walker, M. F. 1961, ApJ, 133, 1081 